\title{
Microbial biosynthesis of lactate esters
}

\author{
Jong-Won Lee ${ }^{1,2}$ and Cong T. Trinh ${ }^{1,2,3^{*}}$ (D)
}

\begin{abstract}
Background: Green organic solvents such as lactate esters have broad industrial applications and favorable environmental profiles. Thus, manufacturing and use of these biodegradable solvents from renewable feedstocks help benefit the environment. However, to date, the direct microbial biosynthesis of lactate esters from fermentable sugars has not yet been demonstrated.

Results: In this study, we present a microbial conversion platform for direct biosynthesis of lactate esters from fermentable sugars. First, we designed a pyruvate-to-lactate ester module, consisting of a lactate dehydrogenase $(I d h A)$ to convert pyruvate to lactate, a propionate CoA-transferase ( $p c t)$ to convert lactate to lactyl-CoA, and an alcohol acyltransferase (AAT) to condense lactyl-CoA and alcohol(s) to make lactate ester(s). By generating a library of five pyruvate-to-lactate ester modules with divergent AATs, we screened for the best module(s) capable of producing a wide range of linear, branched, and aromatic lactate esters with an external alcohol supply. By co-introducing a pyruvate-to-lactate ester module and an alcohol (i.e., ethanol, isobutanol) module into a modular Escherichia coli (chassis) cell, we demonstrated for the first time the microbial biosynthesis of ethyl and isobutyl lactate esters directly from glucose. In an attempt to enhance ethyl lactate production as a proof-of-study, we re-modularized the pathway into (1) the upstream module to generate the ethanol and lactate precursors and (2) the downstream module to generate lactyl-CoA and condense it with ethanol to produce the target ethyl lactate. By manipulating the metabolic fluxes of the upstream and downstream modules through plasmid copy numbers, promoters, ribosome binding sites, and environmental perturbation, we were able to probe and alleviate the metabolic bottlenecks by improving ethyl lactate production by 4.96 -fold. We found that AAT is the most rate-limiting step in biosynthesis of lactate esters likely due to its low activity and specificity toward the non-natural substrate lactyl-CoA and alcohols.
\end{abstract}

Conclusions: We have successfully established the biosynthesis pathway of lactate esters from fermentable sugars and demonstrated for the first time the direct fermentative production of lactate esters from glucose using an E. coli modular cell. This study defines a cornerstone for the microbial production of lactate esters as green solvents from renewable resources with novel industrial applications.

Keywords: Ester, Lactate ester, Ethyl lactate, Isobutyl lactate, Acetate ester, Alcohol acyltransferase, Green solvent, Modular cell, Escherichia coli

\section{Background}

Solvents are widely used as primary components of cleaning agents, adhesives, and coatings and in assisting mass and heat transfer, separation and purification of chemical processes [1]. However, these solvents are volatile organic compounds (VOCs) that contribute to ozone depletion and photochemical smog via free

\footnotetext{
*Correspondence: ctrinh@utk.edu

${ }^{3}$ Department of Chemical and Biomolecular Engineering, University

of Tennessee, 1512 Middle Dr., DO\#432, Knoxville, TN 37996, USA

Full list of author information is available at the end of the article
}

radical air oxidation and hence cause many public health problems such as eye irritation, headache, allergic skin reaction, and cancer $[1,2]$. Thus, recent interest in the use of alternative green solvents is increasing to satisfy environmental regulation and compelling demand for the eco-friendly solvents derived from renewable sources $[3,4]$.

Lactate esters are platform chemicals that have a broad range of industrial applications in flavor, fragrance, and pharmaceutical industries [5]. These esters are generally considered as green solvents because of their favorable 
toxicological and environmental profiles. For instance, ethyl lactate is $100 \%$ biodegradable, non-carcinogenic, non-corrosive, low volatile, and unhazardous to human health and the environment [6]. Due to the unique beneficial properties of ethyl lactate, it has been approved as a Significant New Alternatives Policy (SNAP) solvent by the U.S. Environmental Protection Agency (EPA) and as food additives by the U.S. Food and Drug Administration (FDA) [6]. Recent technical and economic analysis conducted by the National Renewable Energy Laboratory (NREL) considers ethyl lactate to be one of the top 12 bioproducts [7].

In industrial chemical processes, lactate esters are currently produced by esterification of lactic acid with alcohols using homogenous catalysts (e.g., sulfuric acid, hydrogen chloride, and/or phosphoric acid) under high temperature reaction conditions [8]. However, use of strong acids as catalysts causes corrosive problems and often requires more costly equipment for process operation and safety. Furthermore, the esterification reactions are thermodynamically unfavorable $(\Delta G=+5 \mathrm{kcal} / \mathrm{mol})$ in aqueous solutions and often encounter significant challenge due to self-polymerization of lactate [9]. Alternatively, microbial catalysts can be harnessed to produce these esters from renewable and sustainable feedstocks in a thermodynamically favorable reaction $(\Delta G=-7.5 \mathrm{kcal} /$ $\mathrm{mol}$ ) in an aqueous phase environment at room temperature and atmospheric pressure [10-16]. This reaction uses an alcohol acyltransferase (AAT) to generate an ester by condensing an alcohol and an acyl-CoA. AAT can catalyze a broad substrate range including (i) linear or branched short-to-long chain fatty alcohols $[10,11$, 17], (ii) aromatic alcohols [18], and (iii) terpenols [19-22] as well as various fatty acyl-CoAs $[11,13]$. To date, while microbial biosynthesis of the precursor metabolites for lactate esters have been well established such as lactate [13, 16, 23-27], lactyl-CoA [28-30], ethanol [31, 32], propanol [33], isopropanol [34], butanol [35], isobutanol [36], amyl alcohol [37], isoamyl alcohol [38], benzyl alcohol [39], 2-phenylethanol [40, 41], and terpenols [19-22], the direct microbial biosynthesis of lactate esters from fermentable sugars has not yet been demonstrated.

In this work, we aimed to demonstrate the feasibility of microbial production of lactate esters as green organic solvents from renewable resources. To enable the direct microbial biosynthesis of lactate esters from fermentable sugars, we first screened for an efficient AAT suitable for lactate ester production using a library of five pyruvateto-lactate ester modules with divergent AATs. We next demonstrated direct fermentative biosynthesis of ethyl and isobutyl lactate esters from glucose by co-introducing a pyruvate-to-lactate ester module and an alcohol module (i.e., ethanol and isobutanol) into an engineered
Escherichia coli modular cell. As a proof-of-study to improve ethyl lactate production, we employed a combination of metabolic engineering and synthetic biology approaches to dissect the pathway to probe and alleviate the potential metabolic bottlenecks.

\section{Results and discussion}

In vivo screening of efficient AATs critical for lactate ester biosynthesis

The substrate specificity of AATs is critical to produce target esters [13]. For example, ATF1 exhibits substrate preference for biosynthesis of acyl (C4-C6) acetates while SAAT and VAAT prefer biosynthesis of ethyl (C2C6) acylates. Even though both SAAT and VAAT are derived from the same strawberry genus, they also show very distinct substrate preferences; specifically, SAAT prefers longer (C4-C6) acyl-CoAs whereas VAAT prefers shorter (C2-C4) acyl-CoAs. To date, none of AATs have been tested for lactate ester biosynthesis. Thus, to enable lactate ester biosynthesis, we began with identification of the best AAT candidate. We designed, constructed, and characterized a library of five pyruvate-to-lactate ester modules (pJW002-006) carrying five divergent AATs including ATF1, ATF2, SAAT, VAAT, and AtfA, respectively. AtfA was used as a negative control because it prefers long-chain acyl-CoAs (C14-C18) and alcohols (C14-C18) [42]. For characterization, $2 \mathrm{~g} / \mathrm{L}$ of ethanol, propanol, butanol, isobutanol, isoamyl alcohol, and benzyl alcohol were added to culture media with $0.5 \mathrm{mM}$ of IPTG for pathway induction to evaluate biosynthesis of six different lactate esters including ethyl lactate, propyl lactate, butyl lactate, isobutyl lactate, isoamyl lactate, and benzyl lactate, respectively, in high cell density cultures (Fig. 1a).

The results show that most of the strains could produce different types of lactate esters with external supply of alcohols (Fig. 1b, c). EcJW104 achieved the highest titer of lactate esters in all cases, producing $1.59 \pm 0.04 \mathrm{mg} / \mathrm{L}$ of ethyl lactate, $5.46 \pm 0.25 \mathrm{mg} / \mathrm{L}$ of propyl lactate, $11.75 \pm 0.43 \mathrm{mg} / \mathrm{L}$ of butyl lactate, $9.92 \pm 0.08 \mathrm{mg} / \mathrm{L}$ of isobutyl lactate, $24.73 \pm 0.58 \mathrm{mg} / \mathrm{L}$ of isoamyl lactate, and $51.59 \pm 2.09 \mathrm{mg} / \mathrm{L}$ of benzyl lactate in ethanol, propanol, butanol, isobutanol, isoamyl alcohol, and benzyl alcohol doping, respectively. The lactate ester biosynthesis of EcJW104 exhibited different alcohol substrate preference in the following order: benzyl alcohol $>$ isoamyl alcohol $>$ butanol $>$ isobutanol $>$ propanol $>$ ethanol (Fig. 1b, Additional file 1: Table S2).

Due to the presence of endogenous acetyl-CoA, we also produced acetate esters in addition to lactate esters (Fig. 1). Among the strains, EcJW101 achieved the highest titers of acetate esters in all cases, producing $115.52 \pm 4.83 \mathrm{mg} / \mathrm{L}$ of ethyl acetate, $801.62 \pm 33.51 \mathrm{mg} / \mathrm{L}$ of propyl acetate, 


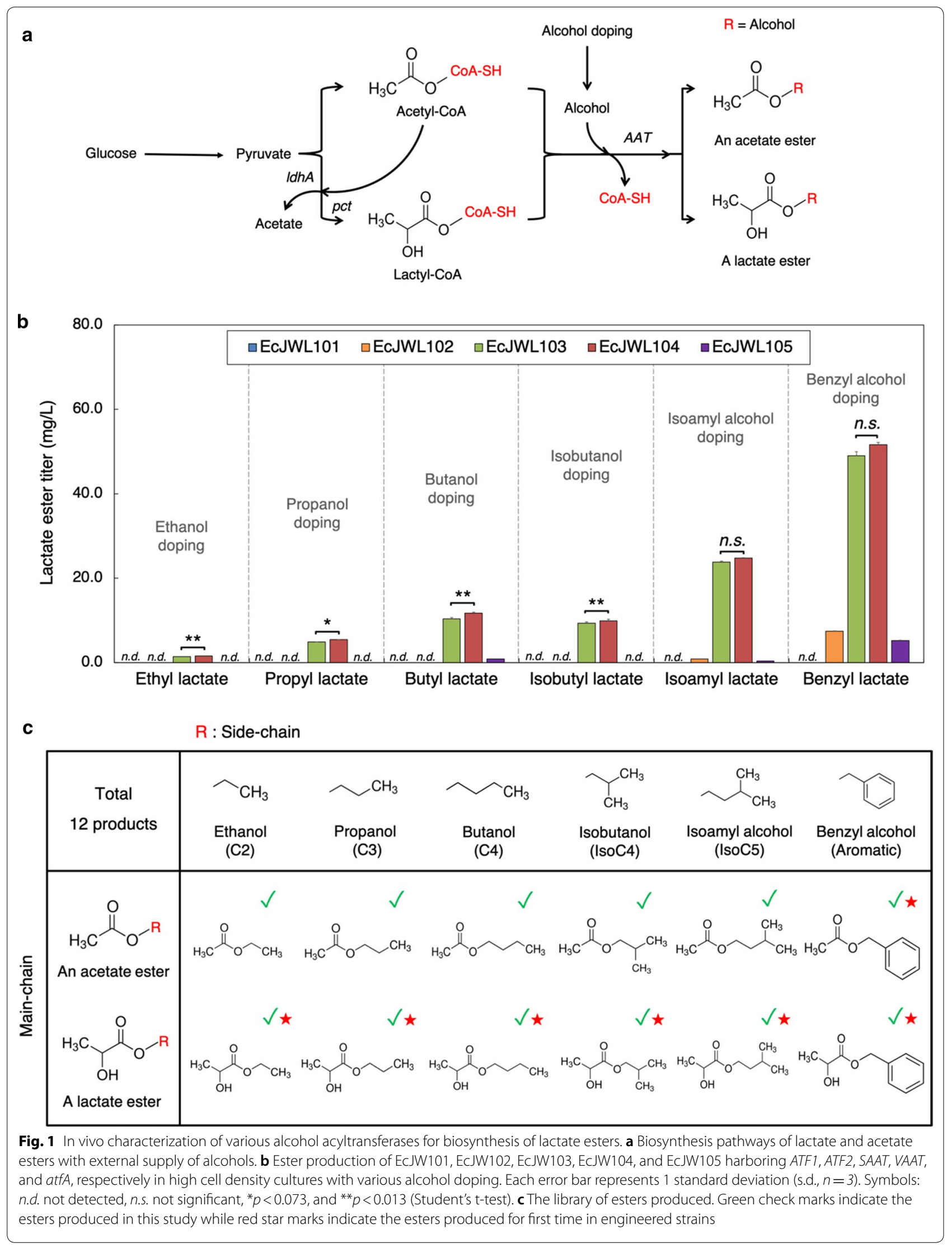


$1017.90 \pm 20.21 \mathrm{mg} / \mathrm{L}$ of butyl acetate, $1210.40 \pm 24.83 \mathrm{mg} / \mathrm{L}$ of isobutyl acetate, $692.73 \pm 7.65 \mathrm{mg} / \mathrm{L}$ of isoamyl acetate, and $1177.98 \pm 45.72 \mathrm{mg} / \mathrm{L}$ of benzyl acetate in ethanol, propanol, butanol, isobutanol, isoamyl alcohol, and benzyl alcohol doping, respectively. EcJW101 showed different alcohol substrate preference for the acetate ester biosynthesis in the following order: isobutanol $>$ benzyl alcohol $>$ butanol $>$ propanol $>$ isoamyl alcohol $>$ ethanol (Additional file 1: Table S2).

Taken altogether, VAAT and ATF1 are the most suitable AATs for biosynthesis of lactate esters and acetate esters, respectively. Among the library of 12 esters (Fig. 1c), seven of these esters, including ethyl lactate, propyl lactate, butyl lactate, isobutyl lactate, isoamyl lactate, benzyl lactate, and benzyl acetate, were demonstrated for in vivo production in microbes for the first time. EcJW104 that harbors the pyruvate-to-lactate module with VAAT could produce 6 out of 6 target lactate esters including ethyl, propyl, butyl, isobutyl, isoamyl, and benzyl lactate. Since EcJW104 achieved the highest titer of lactate esters in all cases, it was selected for establishing the biosynthesis pathway of lactate esters from glucose.

\section{Establishing the lactate ester biosynthesis pathways}

We next demonstrated direct fermentative production of lactate esters from glucose using the best VAAT candidate. We focused on the biosynthesis of ethyl and isobutyl lactate esters. We designed the biosynthesis pathways for ethyl and isobutyl lactate by combining the pyruvate-to-lactate ester module (pJW005) with the ethanol (pCT24) and isobutanol (pCT13) modules, respectively. By co-transforming pJW005/pCT24 and pJW005/pCT13 into the modular cell EcDL002, we generated the production strains, EcJW201 and EcJW202, for evaluating direct conversion of glucose to ethyl and isobutyl lactate esters, respectively.

We characterized EcJW201 and EcJW202 together with the parent strain, EcDL002, as a negative control in high cell density cultures. The results show EcJW201 and EcJW202 produced ethyl (Fig. 2a) and isobutyl (Fig. 2b) lactate from glucose, respectively, while the negative control strain EcDL002 could not. Consistently, the expressions of metabolic enzymes of the ethyl and isobutyl lactate pathways were confirmed in EcJW201 and EcJW202, respectively, by SDS-PAGE analysis (Additional file 2: Figure S1). During $24 \mathrm{~h}$ fermentation, EcJW201 produced $2.24 \pm 0.28 \mathrm{mg} / \mathrm{L}$ of ethyl lactate with a specific productivity of $0.04 \pm 0.00 \mathrm{mg} / \mathrm{gDCW} / \mathrm{h}$ while EcJW202 produced $0.26 \pm 0.01 \mathrm{mg} / \mathrm{L}$ of isobutyl lactate with a specific productivity of $0.01 \pm 0.00 \mathrm{mg} /$ $\mathrm{gDCW} / \mathrm{h}$. In addition to ethyl or isobutyl lactate biosynthesis, EcJW201 also produced $92.25 \pm 9.20 \mathrm{mg} / \mathrm{L}$ of ethyl acetate while EcJW202 generated $1.36 \pm 0.74 \mathrm{mg} / \mathrm{L}$ of ethyl acetate and $0.34 \pm 0.07 \mathrm{mg} / \mathrm{L}$ of isobutyl acetate (Additional file 1: Table S3A). Taken altogether, the direct microbial synthesis of lactate esters from fermentable sugar was successfully demonstrated. Since the lactate ester production was low, the next logical step was to identify and alleviate the key pathway bottlenecks for enhanced lactate ester biosynthesis. As proof-of-principle, we focused on optimization of the ethyl lactate production as presented in the subsequent sections.

\section{Identifying and alleviating key bottlenecks of the ethyl lactate biosynthesis pathway \\ Evaluating the biosynthesis of ethyl lactate in $\mathrm{pH}$-controlled fermentation as a basis to identify potential pathway bottlenecks}

In an attempt to identify the key bottlenecks of the ethyl lactate biosynthesis pathway, we characterized EcJW201 in pH-controlled bioreactors. The results show that EcJW201 produced $9.17 \pm 0.12 \mathrm{mg} / \mathrm{L}$ of ethyl lactate with a specific productivity of $0.15 \pm 0.02 \mathrm{mg} /$ $\mathrm{gDCW} / \mathrm{h}$ and a yield of $0.19 \pm 0.00 \mathrm{mg} / \mathrm{g}$ glucose (Fig. 2c, Additional file 1: Table S3B) in $18 \mathrm{~h}$. Under $\mathrm{pH}$-controlled fermentation, EcJW201 achieved 4.09fold (from $2.24 \pm 0.28$ to $9.17 \pm 0.12 \mathrm{mg} / \mathrm{L}$ ), 3.75-fold (from $0.04 \pm 0.00$ to $0.15 \pm 0.02 \mathrm{mg} / \mathrm{gDCW} / \mathrm{h}$ ), and 19 -fold (from $0.01 \pm 0.00$ to $0.19 \pm 0.00 \mathrm{mg} / \mathrm{g}$ glucose) improvement in titer, specific productivity, and yield, respectively, as compared to the strain performance in the high cell density culture. It is interesting to observe that ethyl acetate was first produced then consumed after $10 \mathrm{~h}$, which is likely due to the endogenous esterase of $E$. coli as observed in a recent study [15]. Different from ethyl acetate, we did not observe ethyl lactate degradation during fermentation, especially after glucose was depleted. Even though the strain performance in $\mathrm{pH}$-controlled bioreactors was enhanced by increased supply of precursor metabolites $(19.35 \pm 0.29 \mathrm{~g} / \mathrm{L}$ of lactate and $10.31 \pm 0.41 \mathrm{~g} / \mathrm{L}$ of ethanol, Additional file 1: Table S3B) from higher concentration of carbon source, the titer of ethyl lactate did not increase during the fermentation. This result suggests that (i) rate-limiting conversion of lactate into lactyl-CoA by Pct and/or condensation of lactyl-CoA with an ethanol by VAAT and/or (ii) toxicity of ethyl lactate on $E$. coli health might have limited lactate ester biosynthesis. Therefore, to enhance ethyl lactate production, it is important to elucidate and alleviate these identified potential bottlenecks.

\section{Ethyl lactate exhibited minimal toxicity on cell growth among lactate esters}

To determine whether lactate esters inhibited cell growth and hence contributed to low lactate ester production, 


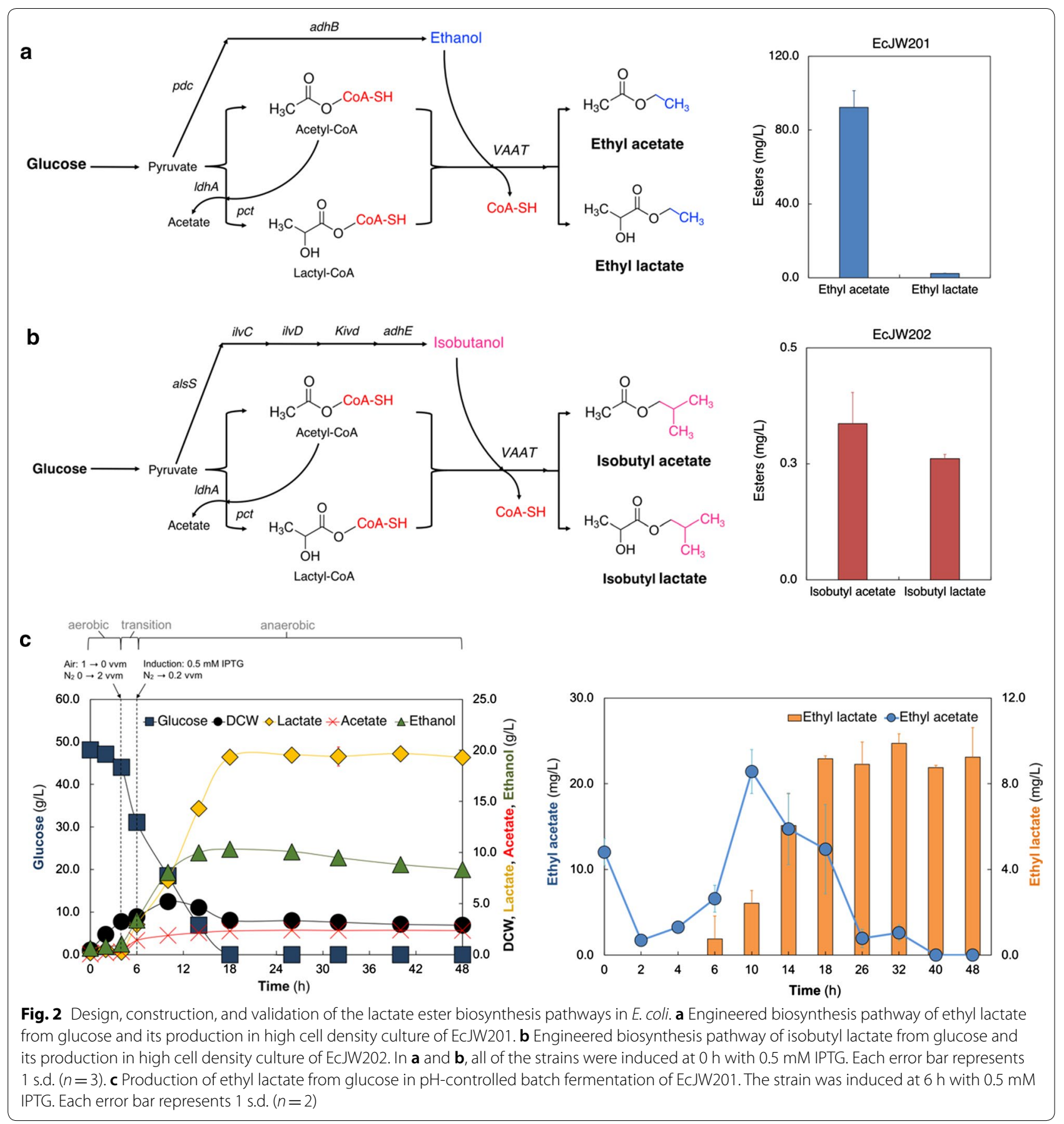

we cultured the parent strain, EcDL002, in a microplate reader with or without supply of various concentrations of lactate esters including ethyl, propyl, butyl, isobutyl, isoamyl, or benzyl lactate. The results show that ethyl lactate was the least toxic among the six lactate esters characterized where the growth rate $(0.47 \pm 0.04 \mathrm{~L} / \mathrm{h})$ and cell titer $\left(\mathrm{OD}_{600}=0.42 \pm 0.03\right)$ decreased by $6 \%$ and $10 \%$, respectively, upon cell exposure to $5 \mathrm{~g} / \mathrm{L}$ ethyl lactate. On the other hand, isoamyl lactate was the most toxic among the lactate esters, where cell exposure to only $0.5 \mathrm{~g} / \mathrm{L}$ ester resulted in $18 \%$ and $15 \%$ reduction in the growth rate $(0.41 \pm 0.02 \mathrm{~L} / \mathrm{h})$ and $\mathrm{OD}_{600}(0.40 \pm 0.03)$, respectively (Additional file 2: Figure S2A). The toxicity of lactate esters can be ranked in the following order: isoamyl lactate $>$ benzyl lactate $>$ butyl lactate $>$ isobutyl lactate $>$ propyl lactate $>$ ethyl lactate. There existed a positive correlation between the $\log \mathrm{P}$ values of lactate esters and their degrees of toxicity (Additional file 2: Figure 
S2B). This result was consistent with literature, illustrating that increasing toxicity of esters is highly correlated with increasing chain length of alcohol moieties that can severely disrupt cell membrane [43]. It should be note that since $E$. coli can effectively secrete short-chain esters [10], external exposure of cells to lactate esters in our experiment design is sufficient to probe the potential toxicity caused by endogenous production of these esters. Taken altogether, ethyl lactate is the least toxic and was not likely the main reason for the low production of ethyl lactate observed. It was likely the downstream pathway, responsible for conversion of lactate into lactyl-CoA by Pct and/or condensation of lactyl-CoA with ethanol by VAAT, might have been contributed to the inefficient ethyl lactate biosynthesis.

Downstream pathway of the lactate ester biosynthesis is the key bottleneck To identify and alleviate the ethyl lactate biosynthesis pathway, we re-modularized it with two new parts: (i) the upstream module carrying $l d h A$, $p d c$, and $a d h B$ for production of lactate and ethanol from sugar and (ii) the downstream module carrying $p c t$ and $V A A T$ for converting lactate into lactyl-CoA and condensing lactyl-CoA and ethanol (Fig. 3a). We controlled metabolic fluxes of these modules by manipulating their plasmid copy numbers and levels of promoter induction with IPTG. By introducing the plasmids pJW007-015 into EcDL002, we generated the strains EcJW106-108 and EcJW203-208, respectively (Fig. 3b). To evaluate the performance of these constructed strains for ethyl lactate production, we characterized them in high cell density cultures induced with various concentrations of IPTG (0.01, 0.1, and $1.0 \mathrm{mM})$.

The results show that EcJW204, carrying the upstream module with a low copy number plasmid (P15A origin) and the downstream module with a high copy number plasmid (RSF1030 origin) induced by $0.01 \mathrm{mM}$ of IPTG, achieved the highest titer of ethyl lactate. As compared to EcJW201, EcJW204 achieved 4.96-fold (an increase from 2.24 to $11.10 \pm 0.58 \mathrm{mg} / \mathrm{L}$ ), 5.50 -fold (from $0.04 \pm 0.00$ to $0.22 \pm 0.02 \mathrm{mg} / \mathrm{gDCW} / \mathrm{h}$ ), and 54.0 -fold (from $0.01 \pm 0.00$ to $0.54 \pm 0.04 \mathrm{mg} / \mathrm{g}$ glucose) improvement in titer, specific productivity, and yield of ethyl lactate, respectively
(Fig. 3b, Additional file 1: Table S5). Upon IPTG induction at $24 \mathrm{~h}$, we observed the reduced cell growth of the host strains with use of high concentration of IPTG (Fig. 3c, Additional file 1: Table S4), suggesting that they suffered from metabolic burden due to overexpression of multiple enzymes [44] and also explaining why use of low concentration of IPTG can help yield better production of ethyl lactate.

Although EcJW204 showed better performance in ethyl lactate production than EcJW201, the accumulation of lactate and ethanol was still observed (Fig. 3f, g, Additional file 1: Table S4), indicating the pathway bottleneck remained. In particular, the downstream module flux was outcompeted by the upstream module flux and hence failed to turn over the precursor metabolites quickly enough. This result helps explain why a combination of the upstream module (for producing lactate and ethanol from sugar) with a low copy number plasmid and the downstream module (for converting lactate into lactyl-CoA and condensing lactyl-CoA and ethanol) with a high copy number plasmid outperformed eight other combinations. Notably, the best ethyl lactate producer EcJW204 achieved the highest lactate and lowest ethanol production among the nine characterized strains (Fig. 3f, g, Additional file 1: Table S4), suggesting redistribution of the carbon flux from ethanol to lactate likely helped improve ethyl lactate production. Thus, we hypothesized that redistribution of the carbon source from ethanol to lactate would help to improve ethyl lactate production. To test this hypothesis, we first examined whether (i) downregulation of the ethanol flux of the upstream module enabled redistribution of the carbon flow from ethanol to lactate and (ii) this redistribution could improve ethyl lactate production before proceeding to investigate the potential bottleneck of downstream module.

High ethanol synthesis of the upstream module was critical for ethyl lactate biosynthesis due to low specificity and activity of AAT To downregulate the ethanol flux of the upstream module, we first reconfigured pJW007, the upstream module of the best performer EcJW204, with a library of two weaker promoters and four weaker synthetic RBSs (Fig. 4a, Additional file 2: Figure S3A),

\footnotetext{
(See figure on next page.)

Fig. 3 Combinatorial modular pathway optimization for enhanced ethyl lactate biosynthesis by varying plasmid copy number. a Re-modularization of the ethyl lactate biosynthesis pathway. Pyruvate-to-lactate ester and ethanol modules were re-modulated into upstream and downstream modules using plasmids with different copy numbers. $\mathbf{b}$ Ethyl lactate production, $\mathbf{c} \mathrm{OD}_{600}$, $\mathbf{d}$ Consumed glucose, e Acetate, $\mathbf{f}$ Lactate, $\mathbf{g}$ Ethanol, and $\mathbf{h}$ Ethyl acetate of EcJW106-108 and EcJW203-208 in high cell density cultures induced with various concentrations of IPTG. Green rectangle: low copy number plasmid (10); P15A: origin of PACYCDuet-1; blue rectangle: medium copy number plasmid (40); ColE1: origin of pETDuet-1; red rectangle: high copy number plasmid (100); RSF1030: origin of pRSFDuet-1; $\mathrm{P}_{\mathrm{T} 7}: \mathrm{T7}$ promoter; $\mathrm{T}_{\mathrm{T}}: \mathrm{T7}$ terminator. All of the strains were induced at $0 \mathrm{~h}$ with $0.01,0.1$, or $1.0 \mathrm{mM} \mathrm{IPTG}$, respectively. Each error bar represents $1 \mathrm{s.d}$. $(n=3)$. Red arrows indicate the selected strain with an optimum concentration of IPTG for the further studies
} 


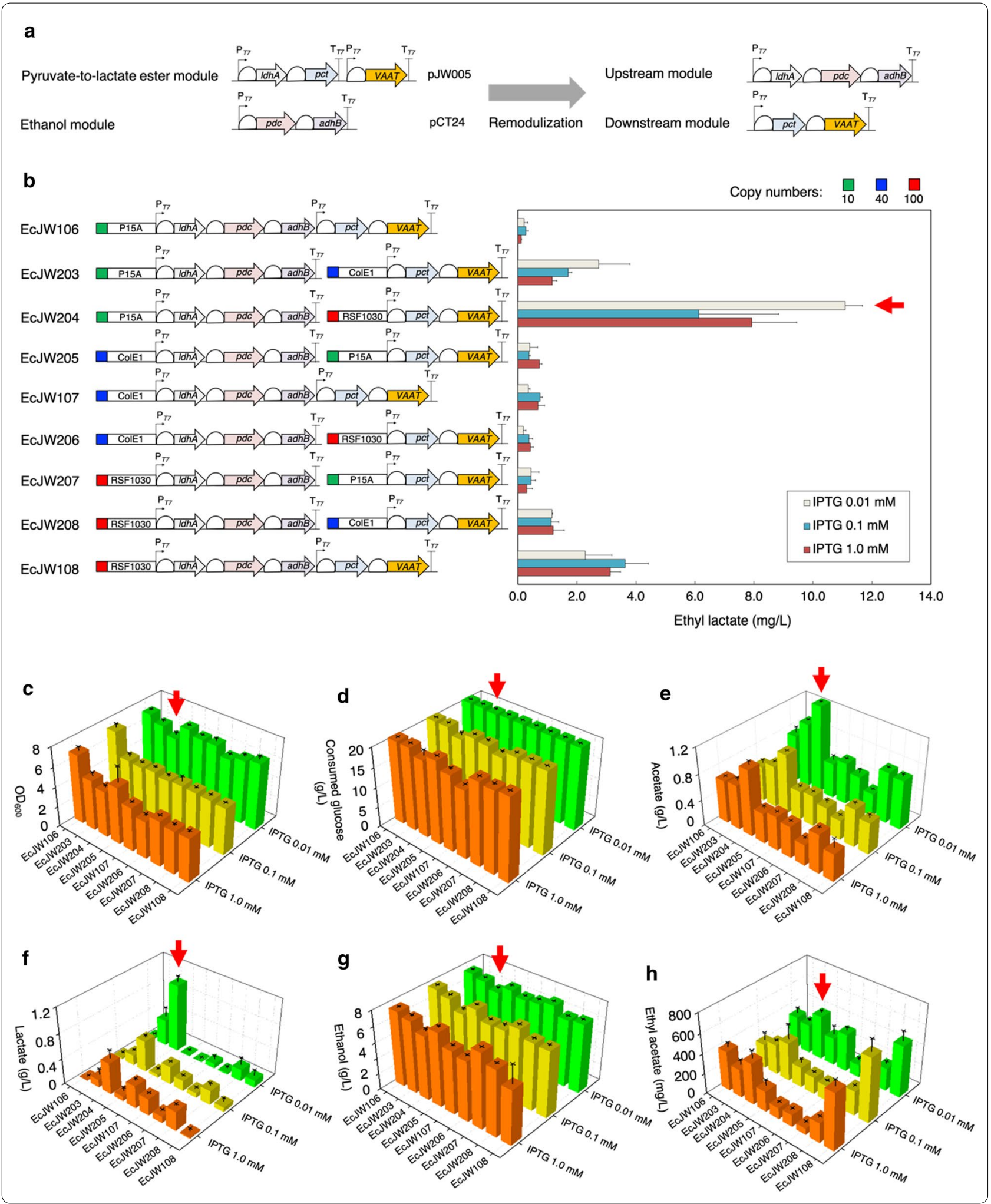


a

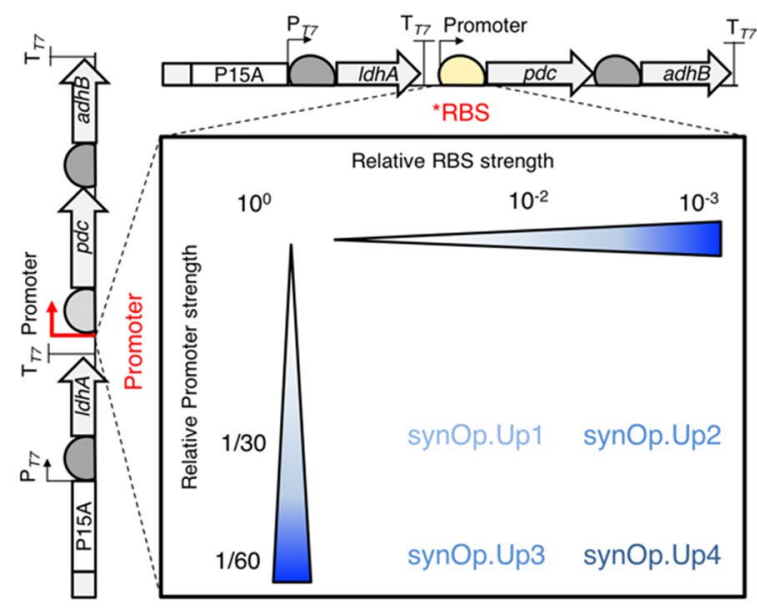

Downstream

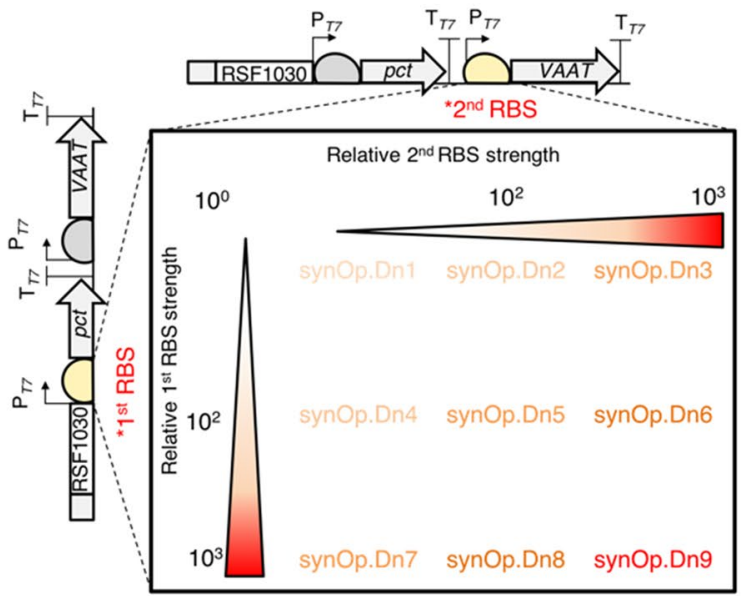

* : Synthetic RBS

b

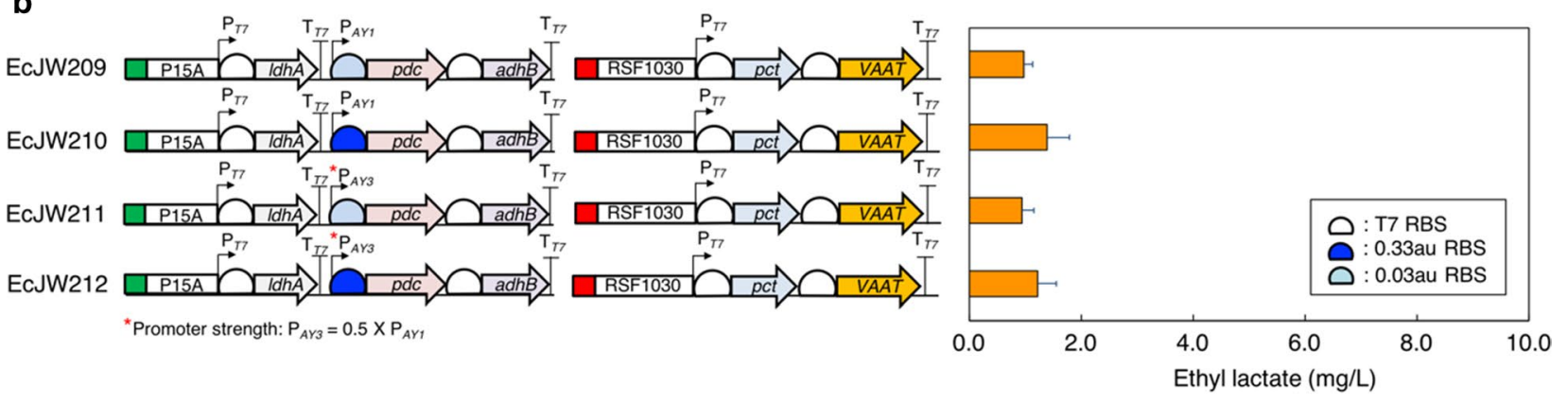

EcJW213 Ec.JW214 EcJW215

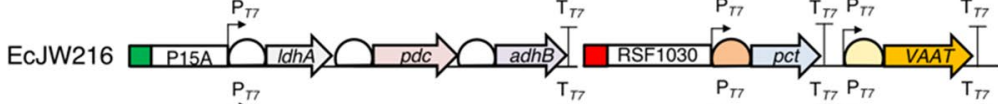

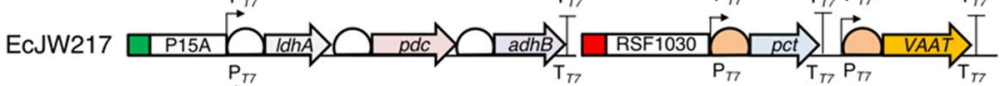
EcJW218 पIP15A

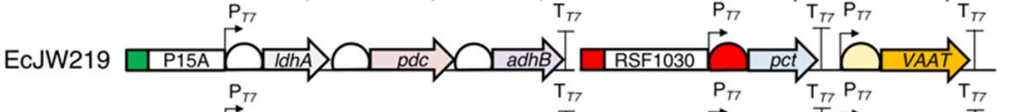
EcJW220

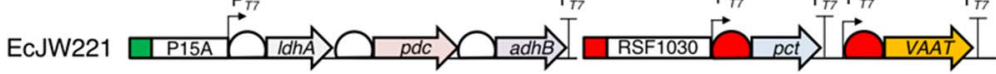

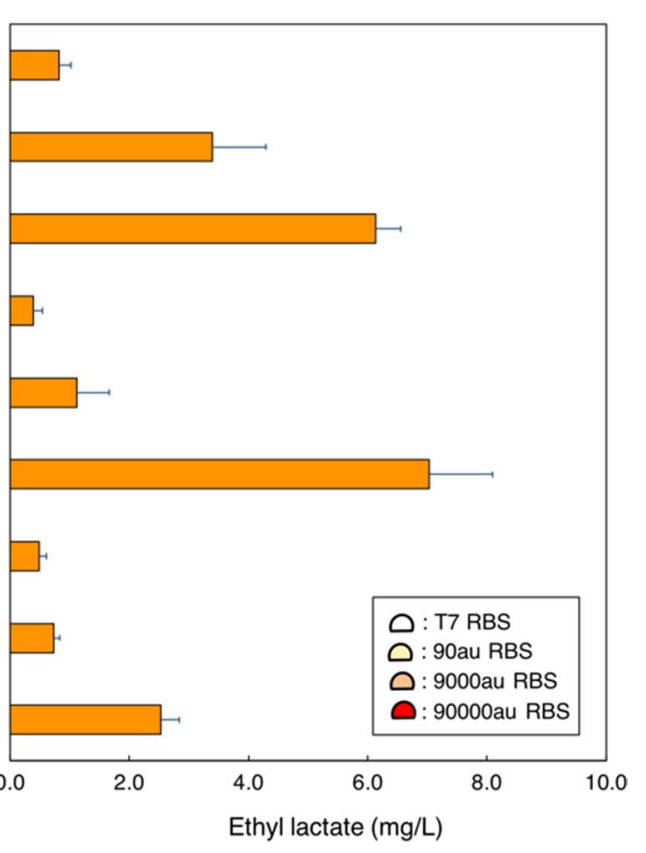


(See figure on previous page.)

Fig. 4 Probing and alleviating the potential metabolic bottlenecks of the upstream or downstream modules of EcJW204 by varying the strength of promoters and/or ribosome binding sites. a Design of synthetic operons for the upstream and downstream modules. For the upstream module, the T7 promoter in MCS2 and the RBS between T7 promoter in MCS2 and the start codon of $p d c$ were replaced with the combination of $P_{A Y 1}$ or $P_{A Y 3}$ promoter and 0.3 or 0.03 a.u. RBS. For the downstream module, the RBS between T7 promoter in MCS1 and the start codon of pct gene and the RBS between T7 promoter in MCS2 and the start codon of VAAT gene were replaced with the combination of 90, 9000, or 90000 a.u. RBS and 90, 9000 , or 90000au RBS, respectively. Production of ethyl lactate in high cell density cultures of $\mathbf{b}$ EcJW209-212 and c EcJW213-221. Green rectangle: low copy number plasmid (10); P15A: origin of pACYCDuet-1; red rectangle: high copy number plasmid (100); RSF1030: origin of pRSFDuet-1; $\mathrm{P}_{\mathrm{T}}$ : T7 promoter; $\mathrm{T}_{\mathrm{T} 7}: \mathrm{T7}$ terminator. All of the strains were induced at $0 \mathrm{~h}$ with $0.01 \mathrm{mM} \mathrm{IPTG}$. Each error bar represents 1 s.d. $(n=3)$

resulting in four new upstream modules (pJW019-022). By introducing each newly constructed upstream module into EcDL002 together with the downstream module pJW012 used in EcJW204, we next generated the strains EcJW209-212 and characterized them in high cell density cultures induced with $0.01 \mathrm{mM}$ IPTG.

The results show that while the carbon flux was successfully redistributed from ethanol to lactate, resulting in 5.97- to 6.92-fold decrease in ethanol production (from $8.30 \pm 0.17$ to $1.39 \pm 0.10 \sim 1.20 \pm 0.01 \mathrm{~g} / \mathrm{L}$ ) and $1.67-$ to 2.59-fold increase in lactate production (from $1.06 \pm 0.09$ to $1.77 \pm 0.37 \mathrm{~g} / \mathrm{L} \sim 2.75 \pm 0.09 \mathrm{~g} / \mathrm{L}$ ) (Additional file 1: Table S6A), the ethyl lactate production was reduced by 7.99 - to 11.81 -fold in ethyl lactate production (from $11.10 \pm 0.58$ to $1.39 \pm 0.40 \sim 0.94 \pm 0.22 \mathrm{mg} / \mathrm{L}$ ) in all four characterized strains as compared to that of EcJW204 (Fig. 4b, Additional file 1: Table S6B). This result suggests that a high level of ethanol is critical for VAAT to produce ethyl lactate.

To support this conclusion, we evaluated the effect of external ethanol supply on production of ethyl esters in high cell density cultures of EcJW209-212 induced with $0.01 \mathrm{mM}$ IPTG. Indeed, with external ethanol supply, we observed enhanced production of both ethyl lactate and ethyl acetate in EcJW209-212. In specific, with addition of $2 \mathrm{~g} / \mathrm{L}$ of ethanol, the ethyl lactate and ethyl acetate production increased by 2.27 - to 3.33 -fold (from $1.39 \pm 0.40$ to $3.15 \pm 0.15 \mathrm{mg} / \mathrm{L} \sim$ from $0.98 \pm 0.15$ to $3.26 \pm 0.26 \mathrm{mg} / \mathrm{L}$ ) and 1.27 - to 2.07 -fold (from $36.46 \pm 3.86$ to $46.22 \pm 1.33 \mathrm{mg} / \mathrm{L} \sim$ from $21.96 \pm 0.84$ to $45.40 \pm 1.20 \mathrm{mg} / \mathrm{L}$ ), respectively (Additional file 1: Table S6). Further addition of ethanol up to $10 \mathrm{~g} / \mathrm{L}$ improved the ethyl lactate and ethyl acetate production by 3.78 - to 5.26 -fold (from $1.39 \pm 0.40$ to $5.26 \pm 0.27 \mathrm{mg} / \mathrm{L} \sim$ from $0.94 \pm 0.15 \mathrm{mg} / \mathrm{L}$ to $4.49 \pm 0.41 \mathrm{mg} / \mathrm{L}$ ) and 4.09 - to 6.92 -fold (from $36.46 \pm 3.86$ to $148.97 \pm 3.80 \mathrm{mg} / \mathrm{L} \sim$ from $21.96 \pm 0.84 \mathrm{mg} / \mathrm{L}$ to $151.87 \pm 2.34 \mathrm{mg} / \mathrm{L}$ ), respectively (Additional file 1: Table S6).
Interestingly, while the total titer of ethyl esters increased with the increasing addition of ethanol (Fig. 5a), the proportion of ethyl lactate in the total ester slightly increased in the range of $3.2-7.0 \%$ (Fig. 5b), suggesting that VAAT prefers acetyl-CoA over lactyl-CoA with ethanol as a co-substrate. Notably, we observed a strong linear correlation between ethyl esters production and the amount of added ethanol (i.e., for ethyl lactate, $R^{2}=0.85-0.94$; for ethyl acetate, $R^{2}=0.99-1.00$ ) (Additional file 2: Figure S4A). The results revealed that abundant availability of ethanol is essential to achieve high production of ethyl esters, indicating the main reason for the improved ethyl lactate production in EcJW204 was most likely due to the upregulation of downstream module with a high copy number plasmid.

AAT was the most rate-limiting step of the downstream module To determine whether Pct for conversion of lactate to lactyl-CoA or VAAT for condensation of lactyl-CoA and an alcohol was the most rate-limiting step of the downstream module, we redesigned and constructed nine downstream modules (pJW027-035) derived from pJW012 of the best performer EcJW204 using a combination of three synthetic RBSs for Pct expression (synRBS ${ }_{\text {pct\#1-3 }}$ ) and three synthetic RBSs for VAAT expression (synRBS ${ }_{\mathrm{VAAT \# 1-3}}$ ) (Fig. 4a, Additional file 2: Figure S3B). We introduced each newly constructed downstream module into EcDL002 together with the original upstream module (pJW007) used in EcJW204 to generate EcJW213-221. Then, we characterized the constructed strains in high cell density cultures induced with $0.01 \mathrm{mM}$ IPTG.

The results show that the strains harboring the stronger RBSs for VAAT expression achieved the higher titers of ethyl lactate and ethyl acetate regardless of the RBS strengths for Pct expression (Fig. 4c, Additional file 1: Table S7). There is a strong linear correlation

(See figure on next page.)

Fig. 5 a Total esters and $\mathbf{b}$ Composition of total esters produced in high cell density cultures of EcJW209-212 with or without addition of ethanol. c Ethyl lactate production of EcJW109-117 with addition of $2 \mathrm{~g} / \mathrm{L}$ of lactate and ethanol. Red rectangle: high copy number plasmid (100); RSF1030: origin of pRSFDuet-1; $\mathrm{P}_{\mathrm{T} 7}: \mathrm{T} 7$ promoter; $\mathrm{T}_{\mathrm{T} 7}: \mathrm{T} 7$ terminator. All of the strains were induced at $0 \mathrm{~h}$ with $0.01 \mathrm{mM}$ IPTG. Each error bar represents $1 \mathrm{~s} . \mathrm{d}$. $(n=3)$ 

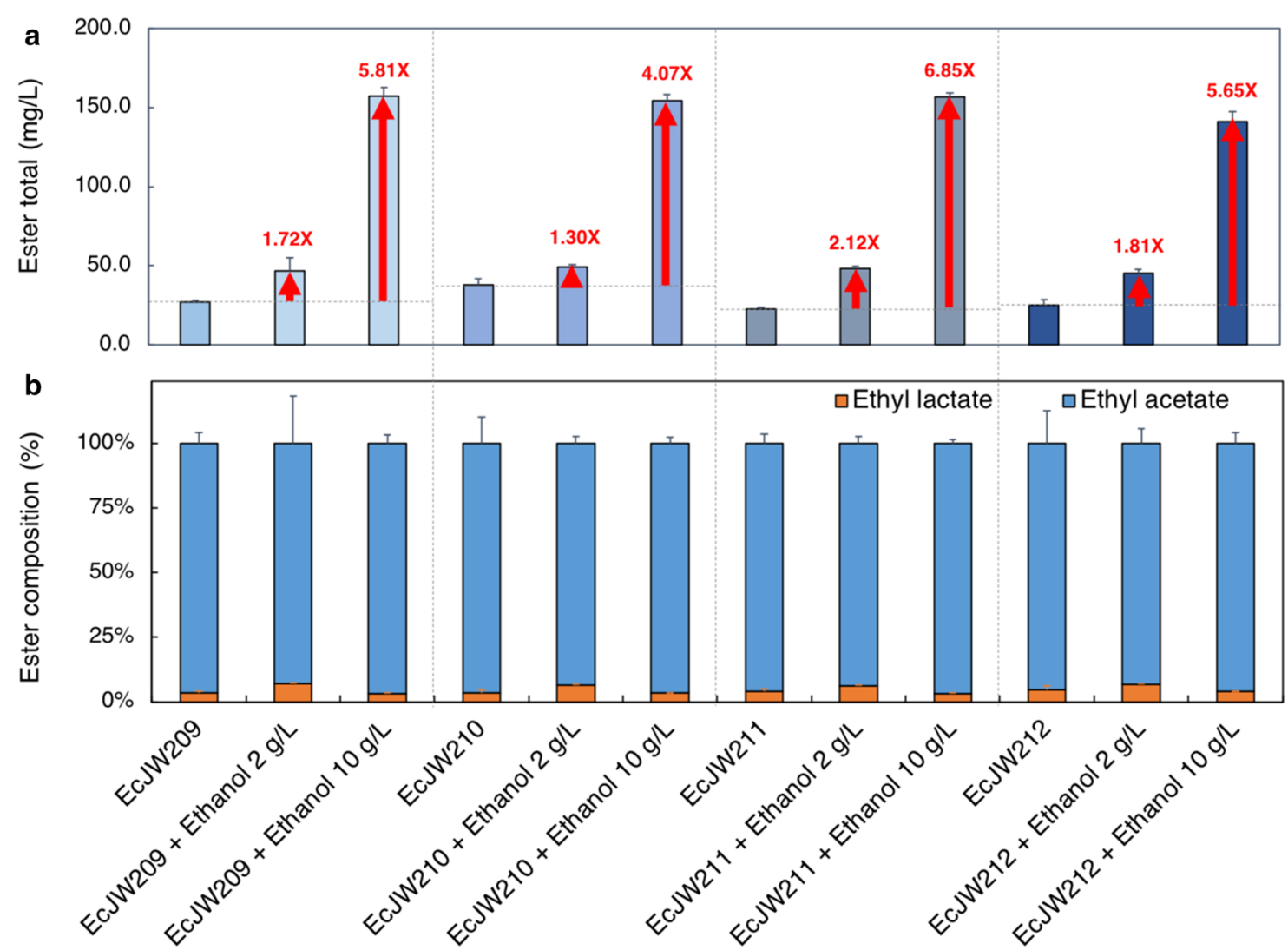

Strains

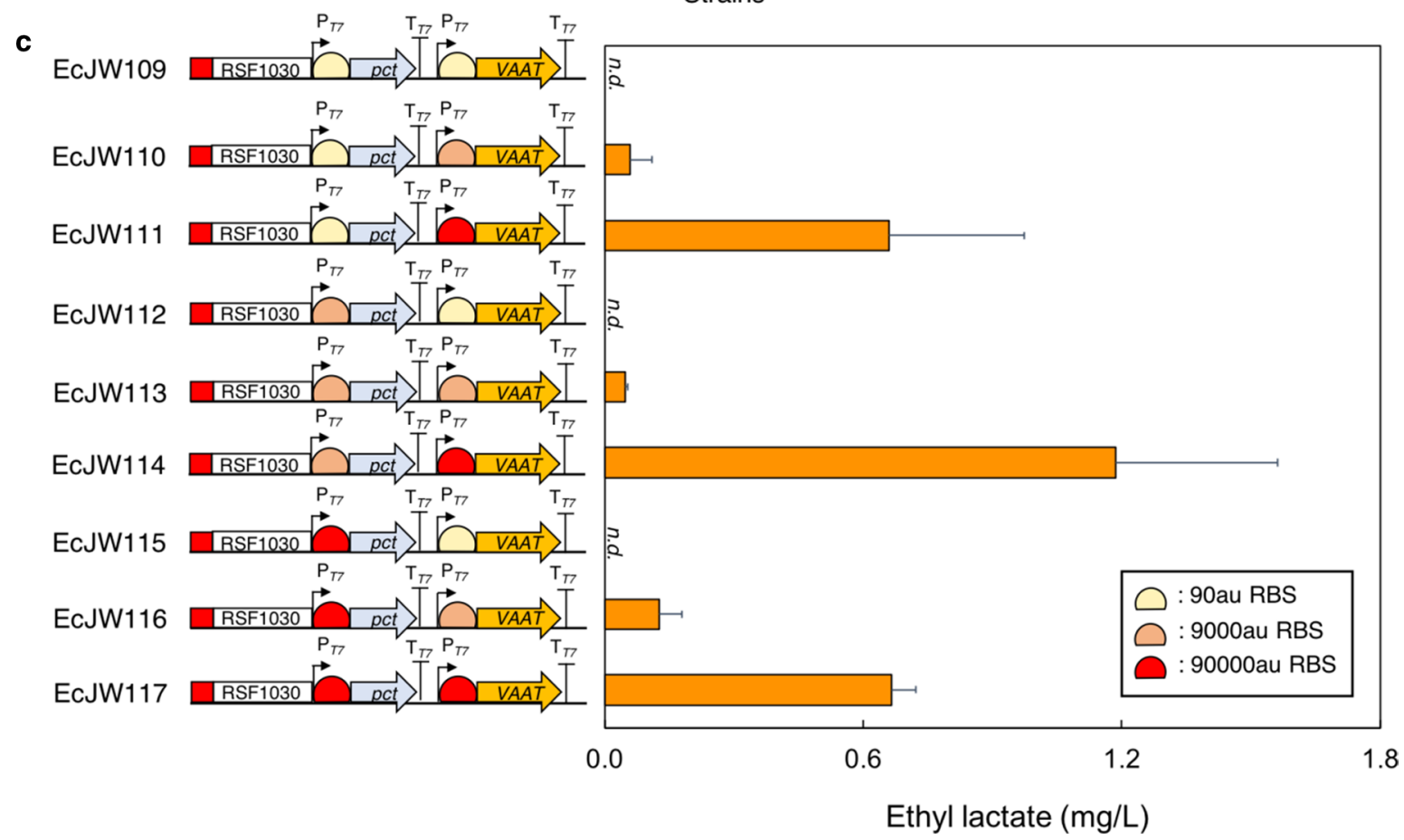


between ethyl ester production and the strength of RBS for VAAT expression (Additional file 2: Figure S4B). To further validate these results without the influence of the upstream module, we additionally constructed the strains EcJW109-117 by introducing nine individual downstream modules (pJW027-035) into EcDL002 and then characterized these strains in high cell density cultures with addition of $2 \mathrm{~g} / \mathrm{L}$ of lactate, $2 \mathrm{~g} / \mathrm{L}$ of ethanol, and $0.01 \mathrm{mM}$ of IPTG. We could observe the same strong linear correlation between ethyl ester production and high VAAT expression without the upstream module (Fig. 5c).

Taken altogether, these results suggest that VAAT not Pct was the most rate-limiting step of the downstream module of the ethyl lactate biosynthesis pathway. In specific, a combination of low affinity toward lactyl-CoA and ethanol of VAAT contributed to low ethyl lactate biosynthesis. Further studies on discovery of novel AATs, exhibiting high activity toward lactyl-CoA and alcohols but not acetyl-CoA, together with rational protein engineering of these enzymes would be warranted for improving lactate ester production.

In principle, the lactate ester platform can be controlled to produce enantiomers with broad industrial applications. Since the endogenous E. coli D-lactate dehydrogenase (LdhA) was overexpressed in the ldhAdeficient modular cell of our study, it is anticipated that $\mathrm{D}-(-)$-lactate and the associated $\mathrm{D}-(-)$-lactate esters were mainly produced. To date, production of optically pure $\mathrm{D}-(-)-[23]$ and $\mathrm{L}-(+)$-form [26] of lactate from glucose in E. coli [25] has been well established. In addition, pct from C. propionicum [28] and Megasphaera elsdenii $[29,30]$ has been used for converting $\mathrm{D}-(-)$-lactate into $\mathrm{D}-(-)$-lactyl-CoA in polylactic acid (PLA) production in E. coli and their catalytic activity toward $\mathrm{L}-(+)$-lactate has also been demonstrated $[45,46]$. Thus, by combining stereospecific Ldh and Pct enzymes together with AATs, it is highly feasible to extend our lactate ester platform for microbial production of stereospecific lactate esters from renewable resources.

\section{Conclusions}

In this study, we have successfully developed a microbial lactate ester production platform and demonstrated for the first time the microbial biosynthesis of lactate esters directly from fermentable sugars in an E. coli modular cell. This study defines a cornerstone for the microbial production of lactate esters as green solvents from renewable resources with novel industrial applications.

\section{Methods}

\section{Strain construction}

The list of strains used in this study is presented in Table 1. For molecular cloning, E. coli TOP10 strain was used. To generate the lactate ester production strains, the modules, including (i) the pyruvate-to-lactate ester modules (pJW002-006), (ii) the upstream and/or downstream modules (pJW007-pJW028), and (iii) the alcohol modules (pCT24 or pCT13), were transformed into the engineered modular E. coli chassis cell, EcDL002 [10] via electroporation [47].

\section{Plasmid construction}

The list of plasmids and primers used in this study are presented in Tables 2 and 3, respectively. Pathway construction includes pyruvate-to-lactate ester modules and a library of upstream and downstream modules with various plasmid copy numbers, promoters, and ribosome binding sites (RBSs).

\section{Construction of pyruvate-to-lactate ester modules}

A library of pyruvate-to-lactate ester modules with five divergent AATs was constructed to screen for an efficient AAT for production of lactate esters via two rounds of cloning. First, the pyruvate-to-lactyl-CoA module (pJW001) was constructed by assembling three DNA fragments: (i) the $l d h A$ gene, encoding D-lactate dehydrogenase, amplified from E. coli MG1655 genomic DNA using the primer pair DL_0032/DL_0033, (ii) the pct gene, encoding propionate CoA-transferase, amplified from Clostridium propionicum genomic DNA using the primer pair DL_0034/DL_0035, and (iii) the backbone amplified from pETite* using the primer pair DL_0001/ DL_0002 [48]. Then, the pyruvate-to-lactate ester modules (pJW002-006) were constructed by assembling three DNA fragments: (i) the pyruvate-to-lactyl-CoA module amplified from pJW001 using the primer pair DL_0032/ DL_0014, (ii) the ATF1 gene amplified from pDL004 for pJW002, the ATF2 gene amplified from pDL005 for pJW003, the SAAT gene amplified from pDL001 for pJW004, the VAAT gene amplified from pDL006 for pJW005, or the atfA gene amplified from pCT16 for pJW006, using the primer pair DL_0015/DL_0016, and (iii) the backbone amplified from pETite* using the primer pair DL_0013/DL_0002. The genes ATF1 and ATF2 are originated from Saccharomyces cerevisiae [49], whereas the genes $S A A T, V A A T$, and atfA are derived from Fragaria ananassa [50], F. vesca [51], and Acinetobacter sp. ADP1 [52], respectively. 
Table 1 A list of strains used in this study

\begin{tabular}{|c|c|c|}
\hline Strains & Genotypes & Sources \\
\hline E. coli TOP10 & $\begin{array}{l}\text { F-mcrA } \triangle \text { (mrr-hsdRMS-mcrBC) } \text { Ф80lacZ } \triangle \mathrm{M} 15 \Delta \text { lacX74 recA1 } \\
\text { araD139 } \triangle \text { (ara leu) } 7697 \text { galU galK rpsL (StrR) endA1 nupG }\end{array}$ & Invitrogen \\
\hline E. coli MG1655 & $\mathrm{F}^{-} \lambda^{-}$ & ATCC 47076 \\
\hline Clostridium propionicum & Wildtype & ATCC 25522 \\
\hline EcDL002 & TCS083 (גDE3) $\triangle$ fadE & {$[10]$} \\
\hline EcJW101 & EcDL002/pJW002; amp ${ }^{R}$ & This study \\
\hline EcJW102 & EcDL002/pJW003; amp ${ }^{R}$ & This study \\
\hline EcJW103 & EcDL002/pJW004; amp ${ }^{R}$ & This study \\
\hline EcJW104 & EcDL002/pJW005; amp ${ }^{R}$ & This study \\
\hline EcJW105 & EcDL002/pJW006; amp ${ }^{R}$ & This study \\
\hline EcJW201 & EcDL002/pJW005 pCT24; amp kan $^{R}$ & This study \\
\hline EcJW202 & EcDL002/pJW005 pCT13; amp ${ }^{R}$ an $^{R}$ & This study \\
\hline EcJW106 & EcDL002/pJW013; $\mathrm{cm}^{R}$ & This study \\
\hline EcJW203 & EcDL002/pJW007 pJW011; cm amp ${ }^{R}$ & This study \\
\hline EcJW204 & EcDL002/pJW007 pJW012; $\mathrm{cm}^{R}$ kan $^{R}$ & This study \\
\hline EcJW205 & EcDL002/pJW008 pJW010; $\mathrm{cm}^{\mathrm{R}} \mathrm{amp} \mathrm{p}^{\mathrm{R}}$ & This study \\
\hline EcJW107 & EcDL002/pJW014;amp ${ }^{R}$ & This study \\
\hline EcJW206 & EcDL002/pJW008 pJW012; amp ${ }^{R}$ ann $^{R}$ & This study \\
\hline EcJW207 & EcDL002/pJW009 pJW010; $\mathrm{cm}^{\mathrm{R}}$ kan $^{\mathrm{R}}$ & This study \\
\hline EcJW208 & EcDL002/pJW009 pJW011; amp ${ }^{R}$ kan $^{R}$ & This study \\
\hline EcJW108 & EcDL002/pJW015; kan ${ }^{R}$ & This study \\
\hline EcJW209 & EcDL002/pJW019 pJW012; $\mathrm{cm}^{\mathrm{R}} \operatorname{kan}^{\mathrm{R}}$ & This study \\
\hline EcJW210 & EcDL002/pJW020 pJW012; $\mathrm{cm}^{\mathrm{R}} \mathrm{kan}^{\mathrm{R}}$ & This study \\
\hline EcJW211 & EcDL002/pJW021 pJW012; $\mathrm{cm}^{\mathrm{R}}$ kan $^{\mathrm{R}}$ & This study \\
\hline EcJW212 & EcDL002/pJW022 pJW012; $\mathrm{cm}^{\mathrm{R}} \mathrm{kan}^{\mathrm{R}}$ & This study \\
\hline EcJW213 & EcDL002/pJW007 pJW027; $\mathrm{cm}^{\mathrm{R}} \mathrm{kan}^{\mathrm{R}}$ & This study \\
\hline EcJW214 & EcDL002/pJW007 pJW028; $\mathrm{cm}^{\mathrm{R}}$ kan $^{\mathrm{R}}$ & This study \\
\hline EcJW215 & EcDL002/pJW007 pJW029; $\mathrm{cm}^{\mathrm{R}} \mathrm{kan}^{\mathrm{R}}$ & This study \\
\hline EcJW216 & EcDL002/pJW007 pJW030; $\mathrm{cm}^{\mathrm{R}} \mathrm{kan}^{\mathrm{R}}$ & This study \\
\hline EcJW217 & EcDL002/pJW007 pJW031; $\mathrm{cm}^{\mathrm{R}} \mathrm{kan}^{\mathrm{R}}$ & This study \\
\hline EcJW218 & EcDL002/pJW007 pJW032; $\mathrm{cm}^{\mathrm{R}} \mathrm{kan}^{\mathrm{R}}$ & This study \\
\hline EcJW219 & EcDL002/pJW007 pJW033; cm kan $^{R}$ & This study \\
\hline EcJW220 & EcDL002/pJW007 pJW034; $\mathrm{cm}^{\mathrm{R}}$ kan $^{\mathrm{R}}$ & This study \\
\hline EcJW221 & EcDL002/pJW007 pJW035; cm kan ${ }^{R}$ & This study \\
\hline EcJW109 & EcDL002/pJW027; kan ${ }^{R}$ & This study \\
\hline EcJW110 & EcDL002/pJW028; kanR & This study \\
\hline EcJW111 & EcDL002/pJW029; kan ${ }^{R}$ & This study \\
\hline EcJW112 & EcDL002/pJW030; kan & This study \\
\hline EcJW113 & EcDL002/pJW031; $\operatorname{kan}^{R}$ & This study \\
\hline EcJW114 & EcDL002/pJW032; kan ${ }^{R}$ & This study \\
\hline EcJW115 & EcDL002/pJW033; kanR & This study \\
\hline EcJW116 & EcDL002/pJW034; kan ${ }^{R}$ & This study \\
\hline EcJW117 & EcDL002/pJW035; kanR & This study \\
\hline
\end{tabular}

\section{Construction of a library of upstream and downstream} modules with various plasmid copy numbers

A library of upstream and downstream modules was constructed to improve ethyl lactate biosynthesis through a combinatorial pathway optimization strategy using three different plasmids: (i) pACYCDuet-1 (P15A origin of replication), (ii) pETDuet-1 (ColE1 origin), and (iii) pRSFDuet-1 (RSF1030 origin), having the plasmid copy numbers of 10,40, and 100, respectively [53]. 
Table $2 \mathrm{~A}$ list of plasmids used in this study

\begin{tabular}{|c|c|c|}
\hline Plasmids & Genotypes & Sources \\
\hline pACYCDuet-1 & Two sets of MCS, $T_{7}$ promoter, P15A ori; $\mathrm{cm}^{\mathrm{R}}$ & Novagen \\
\hline pETDuet-1 & Two sets of MCS, $T_{7}$ promoter, ColE1 ori; amp ${ }^{R}$ & Novagen \\
\hline pRSFDuet-1 & Two sets of MCS, T7 promoter, RSF1030 ori; kan ${ }^{R}$ & Novagen \\
\hline pETite* & $\mathrm{T}_{7}$ promoter, pBR322 ori; $\operatorname{kan}^{\mathrm{R}}$ & {$[10]$} \\
\hline рСТ24 & pETite ${ }^{*} P_{T} ;: p d c:: a d h B:: T_{T 7} ; k^{k} n^{R}$ & [10] \\
\hline pCT13 & pCOLA P $P_{T 7}:: a / s S:: i / v C:: i / v D-P_{T} 7:: k i v d:: a d h E:: T_{T} ; k^{2}{ }^{R}$ & [57] \\
\hline pDL004 & pETite ${ }^{*}$ TF1; kan ${ }^{R}$ & [13] \\
\hline pDL005 & pETite* ATF2; kan ${ }^{\mathrm{R}}$ & [13] \\
\hline pDL001 & pETite* SAAT; kan ${ }^{\mathrm{R}}$ & [13] \\
\hline pDL006 & pETite* VAAT; kan ${ }^{\mathrm{R}}$ & [13] \\
\hline pCT16 & pETite* atfA; kan ${ }^{R}$ & [58] \\
\hline pJW001 & pETite* $P_{T 7}: .1 d h A:: p c t:: T_{T 7} ; a m p^{R}$ & This study \\
\hline pJW002 & pJW001 $\mathrm{P}_{\mathrm{T} 7}::$ IdhA::pct-P $\mathrm{P}_{\mathrm{T} 7}:$ ATF 1:: T T7; amp ${ }^{R}$ & This study \\
\hline pJW003 & 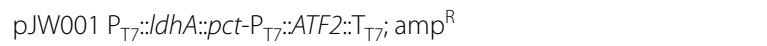 & This study \\
\hline pJW004 & 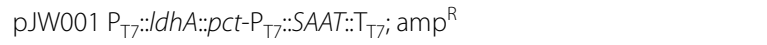 & This study \\
\hline pJW005 & 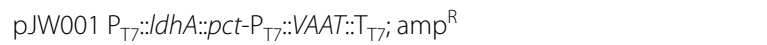 & This study \\
\hline pJW006 & pJW001 $\mathrm{P}_{\mathrm{T} 7}:: / d h A:: p c t-\mathrm{P}_{\mathrm{T} 7}:: a t f A:: \mathrm{T}_{\mathrm{T} 7} ; \mathrm{amp}^{\mathrm{R}}$ & This study \\
\hline pJW007 & pACYCDuet-1 $\mathrm{P}_{\mathrm{T} 7}: . / d h A:: p d c:: a d h B:: T_{T 7} ; \mathrm{cm}^{R}$ & This study \\
\hline pJW008 & 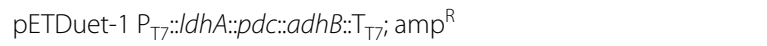 & This study \\
\hline pJW009 & pRSFDuet-1 $\mathrm{P}_{T 7}:: I d h A:: p d c:: a d h B:: \mathrm{T}_{T 7} ; \mathrm{kan}^{\mathrm{R}}$ & This study \\
\hline pJW010 & 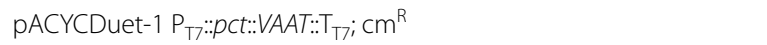 & This study \\
\hline pJW011 & pETDuet-1 $\mathrm{P}_{\mathrm{T} 7}:: p c t:: V A A T:: \mathrm{T}_{\mathrm{T} 7} ; \mathrm{amp}^{\mathrm{R}}$ & This study \\
\hline pJW012 & 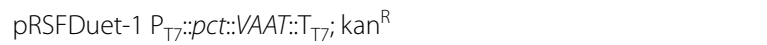 & This study \\
\hline pJW013 & pACYCDuet-1 $\mathrm{P}_{\mathrm{T}} ;: . / d h A:: p d c:: a d h B-\mathrm{P}_{\mathrm{T} 7}: \mathrm{pct}:: V A A T:: \mathrm{T}_{\mathrm{T} 7} ; \mathrm{cm}^{\mathrm{R}}$ & This study \\
\hline pJW014 & pETDuet-1 $\mathrm{P}_{\mathrm{T} 7}:: / d h A:: p d c:: a d h B-\mathrm{P}_{\mathrm{T} 7}:: p c t: \because V A A T:: \mathrm{T}_{T 7} ; \mathrm{amp}^{\mathrm{R}}$ & This study \\
\hline pJW015 & pRSFDuet-1 $\mathrm{P}_{\mathrm{T} 7}:: 1 d h A:: p d c:: a d h B-\mathrm{P}_{\mathrm{T} 7}:: p c t:: V A A T:: \mathrm{T}_{\mathrm{T} 7} ; \mathrm{kan}^{\mathrm{R}}$ & This study \\
\hline pJW016 & pACYCDuet-1 $\mathrm{P}_{\mathrm{T} 7}:: / d h A \cdots: \mathrm{T}_{\mathrm{T} 7}-\mathrm{P}_{\mathrm{T} 7}: \because \mathrm{T}_{\mathrm{T} 7} ; \mathrm{cm}^{\mathrm{R}}$ & This study \\
\hline pJW017 & pACYCDuet-1 $\mathrm{P}_{\mathrm{T} 7}: .1 d h A: \because \mathrm{T}_{\mathrm{T} 7}-\mathrm{P}_{\mathrm{AY} 1}: \because \mathrm{T}_{\mathrm{T}\rangle} ; \mathrm{cm}^{\mathrm{R}}$ & This study \\
\hline pJW018 & pACYCDuet-1 $P_{T}:: 1 d h A: \because T_{T 7}-P_{A Y 3}: \because T_{T 7} ; \mathrm{cm}^{R}$ & This study \\
\hline pJW019 & 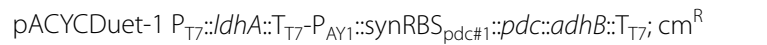 & This study \\
\hline pJW020 & 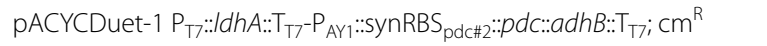 & This study \\
\hline pJW021 & 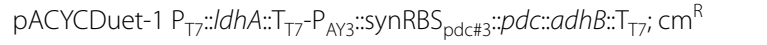 & This study \\
\hline pJW022 & 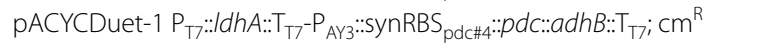 & This study \\
\hline pJW023 & pRSFDuet-1 $\mathrm{P}_{T 7}:: p c t:: \mathrm{T}_{\mathrm{T} 7}-\mathrm{P}_{\mathrm{T} 7}:: \mathrm{T}_{T 7} ; \mathrm{kan}^{\mathrm{R}}$ & This study \\
\hline pJW024 & pRSFDuet-1 $\mathrm{P}_{T 7}::$ SynRBS ${ }_{\text {pct\#1 }}:: p c t:: \mathrm{T}_{T 7}-\mathrm{P}_{\mathrm{T} 7}:: \mathrm{T}_{\mathrm{T} 7} ; \mathrm{kan}^{\mathrm{R}}$ & This study \\
\hline pJW025 & 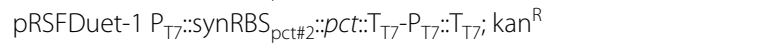 & This study \\
\hline pJW026 & 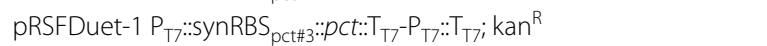 & This study \\
\hline pJW027 & 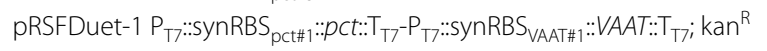 & This study \\
\hline pJW028 & 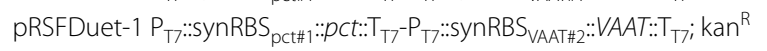 & This study \\
\hline pJW029 & 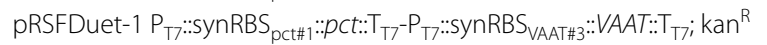 & This study \\
\hline pJW030 & 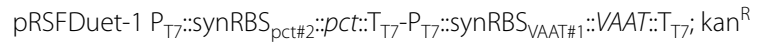 & This study \\
\hline pJW031 & 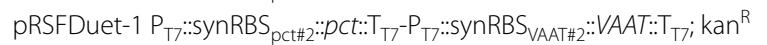 & This study \\
\hline pJW032 & 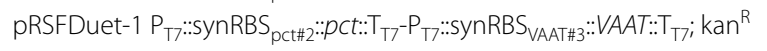 & This study \\
\hline pJW033 & 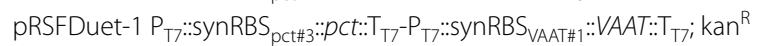 & This study \\
\hline pJW034 & 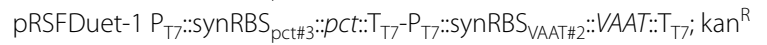 & This study \\
\hline pJW035 & 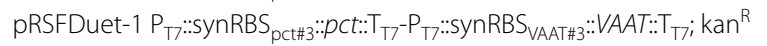 & This study \\
\hline
\end{tabular}


Table 3 A list of primers used in this study

\begin{tabular}{|c|c|}
\hline Primers & Sequences $\left(5^{\prime} \rightarrow 3^{\prime}\right)$ \\
\hline \multicolumn{2}{|c|}{ Pyruvate-to-lactyl-CoA module } \\
\hline DL_0001 & CATCATCACCACCATCACTAA \\
\hline DL_0002 & ATGTATATCTCCTTCTTATAGTTAAAC \\
\hline DL_0032 & TAGAAATAATTTTGTTTAACTATAAGAAGGAGATATACATATGAAACTCGCCGTTTATAG \\
\hline DL_0033 & GGGAACCTTTCTCATTATATCTCCTTTTAAACCAGTTCGTTCGGGC \\
\hline DL_0034 & ACGAACTGGTTTAAAAGGAGATATAATGAGAAAGGTTCCCATTAT \\
\hline DL_0035 & GCCGCTCTATTAGTGATGGTGGTGATGATGTCAGGACTTCATTTCCTTCAG \\
\hline \multicolumn{2}{|c|}{ Pyruvate-to-lactate ester module } \\
\hline DL_0013 & GAGCCTCAGACTCCAGCGTA \\
\hline DL_0014 & ATATCAAGCTTGAATTCGTTACCCGG \\
\hline DL_0015 & GGAGGAACTATATCCGGGTAACGAATTCAAGCTTGATATTAATACGACTCACTATAGGG \\
\hline DL_0016 & GTCCAGTTACGCTGGAGTCTGAGGCTC \\
\hline \multicolumn{2}{|c|}{ Upstream module } \\
\hline JW_0001 & GGGCAGCAGCCATCACCATCATCACCACAGCCAGGATCCATGAAACTCGCCGTTTATAGC \\
\hline JW_0002 & CTAAATAGGTACCGACAGTATAACTCATTATATCTCCTTTTAAACCAGTTCGTTCGGGC \\
\hline JW_0003 & CGAAACCTGCCCGAACGAACTGGTTTAAAAGGAGATATAATGAGTTATACTGTCGGTACC \\
\hline JW_0004 & CGCAAGCTTGTCGACCTGCAGGCGCGCCGAGCTCGAATTCTTAGAAAGCGCTCAGGAAG \\
\hline JW_0005 & GGATCCTGGCTGTGGTGATGA \\
\hline JW_0006 & GAATTCGAGCTCGGCGCG \\
\hline \multicolumn{2}{|c|}{ Downstream module } \\
\hline JW_0007 & GTATATTAGTTAAGTATAAGAAGGAGATATACATATGATGAGAAAGGTTCCCATTATTAC \\
\hline JW_0008 & GAAATTATACTGACCTCAATTTTCTCCATTATATCTCCTTTCAGGACTTCATTTCCTTC \\
\hline JW_0009 & AATGGGTCTGAAGGAAATGAAGTCCTGAAAGGAGATATAATGGAGAAAATTGAGGTCAG \\
\hline JW_0010 & CAAATTTCGCAGCAGCGGTTTCTTTACCAGACTCGAGTCAATATCTTGAAATTAGCGTCT \\
\hline JW_0011 & CATATGTATATCTCCTTCTTATACTTAACT \\
\hline JW_0012 & CTCGAGTCTGGTAAAGAAAC \\
\hline \multicolumn{2}{|c|}{ Synthetic operons for upstream module } \\
\hline JW_0013 & GGGAATTGTGAGCGGATAACAATTCCCCAAGGAGATATAATGAAACTCGCCGTTTATAGC \\
\hline JW_0014 & TTATGCTAGTTATTGCTCAGCGGTGGCGGCCGCTCTATTATTAAACCAGTTCGTTCGG \\
\hline JW_0015 & TCTGGAAAAAGGCGAAACCTGCCCGAACGAACTGGTTTAATAATAGAGCGGCCGC \\
\hline JW_0016 & GATTATGCGGCCGTGTACAATACGATTACTTTCTGTTCGATTTCTACCGAAGAAAGGC \\
\hline JW_0017 & CATTATATCTCCTTGGGGAATTGTTATCCGC \\
\hline JW_0018 & TCGAACAGAAAGTAATCGTATTG \\
\hline JW_0019 & AAATTTGACGGCTAGCTCAGTCCTAGGTACAGTGCTAGCATGAGTTATACTGTCGGTACC \\
\hline JW_0020 & GCGTTCAAATTTCGCAGCAGCGGTTTCTTTACCAGACTCGAGTTAGAAAGCGCTCAGGAA \\
\hline JW_0021 & AAATCTGACAGCTAGCTCAGTCCTAGGTATAATGCTAGCATGAGTTATACTGTCGGTACC \\
\hline JW_0022 & CATGCTAGCACTGTACCTAGGACTGAGCTAGCCGTCAAATTTCGATTATGCGGCC \\
\hline JW_0023 & CATGCTAGCATTATACCTAGGACTGAGCTAGCTGTCAGATTTCGATTATGCGGCC \\
\hline JW_0024 & TACAGTGCTAGCAGCTTAGCGACAACCCTAGGCGCTCGCATGAGTTATACTGTCGGTACC \\
\hline JW_0025 & GTATAATGCTAGCTTAGCAGTACCAGGACGTACCGGAGTATGAGTTATACTGTCGGTACC \\
\hline JW_0026 & TAGGTACAGTGCTAGCACTAGGCCTAGCGATTCCGCTAAATGAGTTATACTGTCGGTACC \\
\hline JW_0027 & TATAATGCTAGCAGTTTACCTAGGGCAATAGCGTACCGAATGAGTTATACTGTCGGTACC \\
\hline JW_0028 & CATGCGAGCGCCTAGGGTTGTCGCTAAGCTGCTAGCACTGTACCTAGG \\
\hline JW_0029 & CATTTAGCGGAATCGCTAGGCCTAGTGCTAGCACTGTACCTAGG \\
\hline JW_0030 & CATACTCCGGTACGTCCTGGTACTGCTAAGCTAGCATTATACCTAGG \\
\hline JW_0031 & CATTCGGTACGCTATTGCCCTAGGTAAACTGCTAGCATTATACCTAGG \\
\hline \multicolumn{2}{|c|}{ Synthetic operons for downstream module } \\
\hline JW_0032 & TTATGCTAGTTATTGCTCAGCGGTGGCGGCCGCTCTATTATCAGGACTTCATTTCCTTCA \\
\hline JW_0033 & TGCAGAAGGCTTAATGGGTCTGAAGGAAATGAAGTCCTGATAATAGAGCGGCCGC \\
\hline
\end{tabular}


Table 3 (continued)

\begin{tabular}{|c|c|}
\hline Primers & Sequences $\left(5^{\prime} \rightarrow 3^{\prime}\right)$ \\
\hline JW_0034 & GATTATGCGGCCGTGTACAATACGATTACTTTCTGTTCGATTTCTACCGAAGAAAGGC \\
\hline JW_0035 & GATATAGCTCGAACGCGGAAAGAGATGAGAAAGGTTCCCATTATTAC \\
\hline JW_0036 & TCAGGACTTCATTTCCTTCA \\
\hline JW_0037 & GCAACCTATTTTAATCCAAGGAAGATCTAATGAGAAAGGTTCCCATTATTAC \\
\hline JW_0038 & GCAATAACAACTAGGAGAGACGACATGAGAAAGGTTCCCATTATTAC \\
\hline JW_0039 & TAATGGGAACCTTTCTCATCTCTTTCCGCGTTCGAGCTATATCGGGGAATTGTTATCCGC \\
\hline JW_0040 & TGCAGAAGGCTTAATGG \\
\hline JW_0041 & GGAACCTTTCTCATTAGATCTTCCTTGGATTAAAATAGGTTGCGGGGAATTGTTATCCGC \\
\hline JW_0042 & TAATGGGAACCTTTCTCATGTCGTCTCTCCTAGTTGTTATTGCGGGGAATTGTTATCCGC \\
\hline JW_0043 & TAACCAAAACACTAACGCAAGATGGAGAAAATTGAGGTCAGT \\
\hline JW_0044 & AGGGCACGAGGAGGAACCAGTAGAATGGAGAAAATTGAGGTCAGT \\
\hline JW_0045 & GCAACCAACACAACGAGGAGGCATTTAATGGAGAAAATTGAGGTCAGT \\
\hline JW_0046 & TACTGACCTCAATTTTCTCCATCTTGCGTTAGTGTTTTGGTTAGGGGAATTGTTATCCGC \\
\hline JW_0047 & CTCAATTTTCTCCATTCTACTGGTTCCTCCTCGTGCCCTGGGGAATTGTTATCCGC \\
\hline JW_0048 & CTCAATTTTCTCCATTAAATGCCTCCTCGTTGTGTTGGTTGCGGGGAATTGTTATCCGC \\
\hline
\end{tabular}

The upstream modules (pJW007-009) were constructed by assembling three DNA fragments: (i) the $l d h A$ gene amplified from pJW001 using the primer pair JW_0001/ JW_0002, (ii) the ethanol module containing $p d c$ and $a d h B$ genes amplified from pCT24 using the primer pair JW_0003/JW_0004, and (iii) the backbone amplified from pACYCDuet-1 for pJW007, from pETDuet-1 for pJW008, or from pRSFDuet-1 for pJW009 using the primer pair JW_0005/JW_0006.

The downstream modules (pJW010-012) were constructed by assembling three DNA fragments: (i) the pct gene amplified from pJW001 using the primer pair JW_0007/JW_0008, (ii) the VAAT gene amplified from pJW005 using the primer pair JW_0009/JW_0010, and (iii) the backbone amplified from pACYCDuet-1 for pJW010, pETDuet-1 for pJW011, or pRSFDuet-1 for pJW012 using the primer pair JW_0011/JW_0012.

The combined upstream and downstream modules (pJW013-015) were constructed by assembling two DNA fragments: (i) the upstream module amplified from pJW007 using the primer pair JW_0001/JW_0004 and (ii) the backbone containing the downstream module amplified from pJW010 for pJW013, pJW011 for pJW014, or pJW012 for pJW015 using the primer pair JW_0005/ JW_0006.

\section{Construction of a library of upstream and downstream modules with various promoters and RBSs}

For tighter regulation of biosynthetic pathway of ethyl lactate, we constructed the upstream and downstream modules with tunable promoters and RBSs.

The upstream modules (pJW019-022) were constructed via three rounds of cloning. First, the $\mathrm{T} 7$ terminator $\left(\mathrm{T}_{T 7}\right)$ was added between the multiple cloning site 1 (MCS1) and MCS2 of the pACYCDuet-1 backbone to create the first intermediate plasmid, pJW016, by assembling three DNA fragments: (i) the $l d h A$ gene amplified from pJW001 using the primer pair JW_0013/JW_0014, (ii) the linker containing $\mathrm{T}_{T 7}$ terminator amplified from pETite* using the primer pair JW_0015/JW_0016, and (iii) the backbone amplified from pACYCDuet-1 using the primer pair JW_0017/JW_0018. Next, the original T7 promoter $\left(\mathrm{P}_{T 7}\right)$ in MCS2 of pJW016 was replaced with the $\mathrm{P}_{A Y 1}\left(\mathrm{BBa} \_\mathrm{J} 23100\right)$ promoter and $\mathrm{P}_{A Y 3}(\mathrm{BBa} 23108)$ promoter to generate two second-intermediate plasmids, pJW017 and pJW018, respectively, by assembling two DNA fragments: (i) the ethanol module amplified from pCT24 under the $\mathrm{P}_{A Y 1}$ promoter for $\mathrm{pJW} 017$ or $\mathrm{P}_{A Y 3}$ promoter for pJW018 using the primer pair JW_0019/ JW_0020 or JW_0021/JW_0020, respectively, and (ii) the backbone amplified from pJW016 using the primer pair JW_0022/JW_0012 or JW_0023/JW_0012, respectively. Last, the final four synthetic operons (pJW019-022) were constructed by assembling two DNA fragments: (i) the ethanol module amplified from pCT24 with the synthetic RBS sequences with predicted translation initiation rates of 0.33 a.u. for pJW019 and pJW021 and 0.03 a.u. for pJW020 and pJW022 using the primer pairs JW_0024/ JW_0020，JW_0025/JW_0020， JW_0026/JW_0020, and JW_0027/JW_0020, respectively, and (ii) the backbone amplified from pJW017 for pJW019, pJW017 for pJW020, pJW018 for pJW021, and pJW018 for pJW022 using the primer pairs JW_0028/JW_0012, JW_0029/ JW_0012, JW_0030/JW_0012, and JW_0031/JW_0012, respectively. The $\mathrm{P}_{A Y 1}$ and $\mathrm{P}_{A Y 3}$ promoter sequences were obtained from the iGEM Anderson promoter 
library (http://parts.igem.org/Promoters/Catalog/Ander son) and the strength of promoters were assigned as $\mathrm{P}_{A Y 3}=0.5 \times \mathrm{P}_{A Y 1}$. The RBS Calculator v2.0 [54, 55] was used to generate four synthetic RBS sequences with predicted translation initiation rates of 0.33 and 0.03 between the $\mathrm{P}_{A Y 1}$ (or $\mathrm{P}_{A Y 3}$ ) promoter and $p d c$ start codon (Additional file 2: Figure S3).

The downstream modules (pJW027-035) were constructed via three rounds of cloning. First, the $T_{T 7}$ terminator was added between MCS1 and MCS2 of the pRSFDuet-1 backbone to generate the first intermediate plasmid, pJW023, by assembling three DNA fragments: (i) the pct gene amplified from pJW001 using the primer pair JW_0013/JW_0032, (ii) the linker containing $\mathrm{T}_{T 7}$ terminator from pETite* using the primer pair JW_0033/ JW_0034, and (iii) the backbone from pRSFDuet-1 using the primer pair JW_0017/JW_0018. Then, the original RBS in MCS1 of pJW023 was replaced with synthetic RBSs of various strengths to generate the second-intermediate plasmids, pJW024-026, by assembling two DNA fragments: (i) the pct gene amplified from pJW001 with the synthetic RBS sequences with predicted translation initiation rates at 90, 9000, or 90000 a.u. for pJW024, pJW025, or pJW026 using the primer pair JW_0035/ JW_0036, JW_0037/JW_0036, or JW_0038/JW_0036, respectively, and (ii) the backbone amplified from pJW023 using the primer pair JW_0039/JW_0040 for pJW024, JW_0041/JW_0040 for pJW025, or JW_0042/ JW_0040 for pJW026, respectively. Last, the final nine downstream modules (pJW027-035) were constructed by assembling a combination of two DNA fragments: (i) the $V A A T$ gene amplified from pDL006 with the synthetic RBS sequences predicted with translation initiation rates of 90,9000 , or 90000 a.u. for pJW027/pJW030/pJW033, pJW028/pJW031/pJW034, or pJW029/pJW032/pJW035 using the primer pair JW_0043/JW_0010, JW_0044/ JW_0010, or JW_0045/JW_0010, respectively, and (ii) the backbone amplified from pJW024, pJW025, or pJW026 for pJW027-029, pJW030-032, or pJW033-035 using the primer pair JW_0046/JW_0012, JW_0047/JW_0012, or JW_0048/JW_0012, respectively. The RBS Calculator v2.0 $[54,55]$ was used to generate six synthetic RBS sequences with predicted translation initiation rates of 90, 9000, and 90000 a.u. between the $\mathrm{P}_{T 7}$ promoter and pct (or VAAT) start codon (Additional file 2: Figure S3).

\section{Culture media and conditions Culture media}

For molecular cloning, seed cultures, and protein expression analysis, the Luria-Bertani (LB) medium, comprising $10 \mathrm{~g} / \mathrm{L}$ peptone, $5 \mathrm{~g} / \mathrm{L}$ yeast extract, and $5 \mathrm{~g} / \mathrm{L} \mathrm{NaCl}$, was used. For high cell density cultures, pre-cultures of bioreactor batch fermentations, and growth inhibition analysis of lactate esters, the M9 hybrid medium [10] with $20 \mathrm{~g} / \mathrm{L}$ glucose was used. For bioreactor batch fermentations, the M9 hybrid medium with $50 \mathrm{~g} / \mathrm{L}$ glucose and $100 \mu \mathrm{L}$ of antifoam (Antifoam 204, Sigma-Aldrich, MO, USA) was used. $30 \mu \mathrm{g} / \mathrm{mL}$ chloramphenicol $(\mathrm{cm})$, $50 \mu \mathrm{g} / \mathrm{mL}$ kanamycin (kan), and/or $50 \mu \mathrm{g} / \mathrm{mL}$ ampicillin (amp) were added to the media for selection where applicable.

\section{High cell density cultures}

For seed cultures, $2 \%(\mathrm{v} / \mathrm{v})$ of stock cells were grown overnight in $5 \mathrm{~mL}$ of LB with appropriate antibiotics. For pre-cultures, $1 \%(\mathrm{v} / \mathrm{v})$ of seed cultures was transferred into $100 \mathrm{~mL}$ of $\mathrm{LB}$ medium in $500-\mathrm{mL}$ baffled flasks. For main cultures, pre-cultures were aerobically grown overnight (at $37^{\circ} \mathrm{C}, 200 \mathrm{rpm}$ ), centrifuged (4700 rpm, $10 \mathrm{~min}$ ), and resuspended to yield an optical density measured at $600 \mathrm{~nm}\left(\mathrm{OD}_{600 \mathrm{~nm}}\right)$ of 3 in M9 hybrid medium containing appropriate concentration of isopropyl-beta-D-thiogalatopyranoside (IPTG) and antibiotics. The resuspended cultures were distributed into $15-\mathrm{mL}$ polypropylene centrifuge tubes (Thermo Scientific, IL, USA) with a working volume of $5 \mathrm{~mL}$ and grown for $24 \mathrm{~h}$ (h) on a $75^{\circ}$ angled platform in a New Brunswick Excella E25 at $37^{\circ} \mathrm{C}, 200 \mathrm{rpm}$. The tubes were capped to generate anaerobic condition. All high cell density culture studies were performed in biological triplicates.

\section{pH-controlled bioreactor batch fermentations}

$\mathrm{pH}$-controlled bioreactor batch fermentations were conducted with a Biostat B+ (Sartorius Stedim, NY, USA) dual 1.5-L fermentation system at a working volume of 1 L M9 hybrid medium. The seed and precultures were prepared as described in high cell density cultures in LB and M9 hybrid media, respectively. For main cultures, $10 \%(\mathrm{v} / \mathrm{v})$ of pre-cultures were inoculated into fermentation cultures. During the fermentation, to achieve high cell density, dual-phase fermentation approach $[25,56]$, aerobic cell growth phase followed by anaerobic production phase, was applied. For the first aerobic phase, the temperature, agitation, and air flow rate were maintained at $37^{\circ} \mathrm{C}, 1000 \mathrm{rpm}$, and 1 volume/volume/min (vvm) for $4 \mathrm{~h}$, respectively. Then, the oxygen in the medium was purged by sparing nitrogen gas at $2 \mathrm{vvm}$ for $2 \mathrm{~h}$ to generate anaerobic condition. For the subsequent anaerobic phase, $0.5 \mathrm{mM}$ of IPTG was added to induce the protein expression, and the culture temperature and nitrogen flow rate were maintained at $30{ }^{\circ} \mathrm{C}$ and $0.2 \mathrm{vvm}$, respectively. During the fermentation, the $\mathrm{pH}$ was maintained at 7.0 with $5 \mathrm{M} \mathrm{KOH}$ and $40 \% \mathrm{H}_{3} \mathrm{PO}_{4}$. Bioreactor batch fermentation studies were performed in biological duplicates. 


\section{Growth inhibition analysis of lactate esters}

Seed cultures of EcDL002 were prepared as described in high cell density cultures. $4 \%(\mathrm{v} / \mathrm{v})$ of seed cultures was inoculated into $100 \mu \mathrm{L}$ of the M9 hybrid media, containing various concentrations $(0.5-40 \mathrm{~g} / \mathrm{L})$ of lactate esters including ethyl-, propyl-, butyl-, isobutyl-, isoamyl-, or benzyl lactate, in a 96-well microplate. Then, the microplate was sealed with a plastic adhesive sealing film, SealPlate ${ }^{\circledR}$ (EXCEL Scientific, Inc., CA, USA) to prevent evaporation of lactate esters and incubated at $37{ }^{\circ} \mathrm{C}$ with continuous shaking using a BioTek Synergy HT microplate reader (BioTek Instruments, Inc., VT, USA). $\mathrm{OD}_{600 \mathrm{~nm}}$ was measured at 20-min intervals. Growth inhibition studies of lactate esters were performed twice in biological triplicates $(n=6)$.

\section{Protein expression and SDS-PAGE analysis}

Seed cultures were prepared as described in high cell density cultures. $1 \%(\mathrm{v} / \mathrm{v})$ of seed cultures subsequently inoculated in 500-mL baffled flasks containing $100 \mathrm{~mL}$ of LB medium. Cells were aerobically grown at $37{ }^{\circ} \mathrm{C}$ and $200 \mathrm{rpm}$ and induced at an $\mathrm{OD}_{600 \mathrm{~nm}}$ of $0.6-0.8$ with $0.5 \mathrm{mM}$ of IPTG. After $4 \mathrm{~h}$ of induction, cells were collected by centrifugation and resuspended in $100 \mathrm{mM}$ of sodium phosphate buffer $(\mathrm{pH} 7.0)$ at the final $\mathrm{OD}_{600 \mathrm{~nm}}$ of 10. Cell pellets were disrupted using a probe-type sonicator (Model 120, Fisher Scientific, NH, USA) on ice-water mixture. The resulting crude extracts were mixed with $6 \times$ sodium dodecyl sulfate (SDS) sample buffer, heated at $100{ }^{\circ} \mathrm{C}$ for $5 \mathrm{~min}$, and then analyzed by SDS-polyacrylamide gel electrophoresis (SDS-PAGE, $14 \%$ polyacrylamide gel). Protein bands were visualized with Coomassie Brilliant Blue staining.

\section{Analytical methods \\ Determination of cell concentrations}

The optical density was measured at $600 \mathrm{~nm}$ using a spectrophotometer (GENESYS 30, Thermo Scientific, IL, USA). The dry cell mass was obtained by multiplication of the optical density of culture broth with a pre-determined conversion factor, $0.48 \mathrm{~g} / \mathrm{L} / \mathrm{OD}$.

\section{High-performance liquid chromatography (HPLC)}

Glucose, lactate, acetate, ethanol, isobutanol, isoamyl alcohol, and benzyl alcohol were quantified using the Shimadzu HPLC system (Shimadzu Inc., MD, USA) equipped with the Aminex HPX-87H cation exchange column (BioRad Inc., CA, USA) heated at $50^{\circ} \mathrm{C}$. A mobile phase of $10 \mathrm{mN} \mathrm{H}_{2} \mathrm{SO}_{4}$ was used at a flow rate of $0.6 \mathrm{~mL} /$ $\mathrm{min}$. Detection was made with the reflective index detector (RID) and UV detector (UVD) at $220 \mathrm{~nm}$.
Gas chromatography coupled with mass spectroscopy (GC) MS)

All esters were quantified by GC/MS. For GC/MS analysis, analytes in the supernatants were extracted with dichloromethane (DCM), containing pentanol as an internal standard, in a $1: 1(\mathrm{v} / \mathrm{v})$ ratio for $1 \mathrm{~h}$ at $37^{\circ} \mathrm{C}$, $200 \mathrm{rpm}$ in $15-\mathrm{mL}$ polypropylene centrifuge tubes. After extraction, supernatant-DCM mixtures were centrifuged and $5 \mu \mathrm{L}$ of DCM extracts were injected into a gas chromatograph (GC) HP 6890 equipped with the mass selective detector (MS) HP 5973. For the GC system, helium was used as the carrier gas at a flow rate of $0.5 \mathrm{~mL} / \mathrm{min}$ and the analytes were separated on a Phenomenex ZB-5 capillary column $(30 \mathrm{~m} \times 0.25 \mathrm{~mm} \times 0.25 \mu \mathrm{m})$. The oven temperature was programmed with an initial temperature of $50{ }^{\circ} \mathrm{C}$ with a $1{ }^{\circ} \mathrm{C} / \mathrm{min}$ ramp up to $58{ }^{\circ} \mathrm{C}$. Next, a $25{ }^{\circ} \mathrm{C} / \mathrm{min}$ ramp was deployed to $235{ }^{\circ} \mathrm{C}$ and then finally held a temperature of $300{ }^{\circ} \mathrm{C}$ for $2 \mathrm{~min}$ to elute any residual non-desired analytes. The injection was performed using a splitless mode with an initial injector temperature of $280{ }^{\circ} \mathrm{C}$. For the MS system, a selected ion monitoring (SIM) mode was deployed to detect analytes.

The SIM parameters for detecting lactate esters were as follows: (i) for pentanol, ions 53.00, 60.00, and 69.00 detected from 5.00 to $7.70 \mathrm{~min}$, (ii) for ethyl lactate, ions 46.00, 47.00, and 75.00 detected from 7.70 to $10.10 \mathrm{~min}$, (iii) for propyl lactate, ions 59.00, 88.00, and 89.00 detected from 10.10 to $11.00 \mathrm{~min}$, (iv) for isobutyl lactate, ions 56.00, 57.00, and 59.00 detected from 11.00 to $11.60 \mathrm{~min}$, (v) for butyl lactate, ions $75.00,91.00$, and 101.00 detected from 11.60 to $12.30 \mathrm{~min}$, (vi) for isoamyl lactate, ions $46.00,73.00,75.00$ from 12.30 to $14.50 \mathrm{~min}$, and (vii) for benzyl lactate, ions $45.00,91.00$, and 180.00 from 14.50 to $15.08 \mathrm{~min}$. The SIM parameters for detecting acetate esters were as follows: (i) for ethyl acetate, ions 45.00, 61.00, and 70.00 detected from 4.22 to $5.35 \mathrm{~min}$, (ii) for propyl acetate, ions 57.00 , 59.00, and 73.00 detected from 5.35 to $6.40 \mathrm{~min}$, (iii) for pentanol, ions 53.00, 60.00, and 69.00 detected from 6.40 to $6.60 \mathrm{~min}$, (iv) for isobutyl acetate, ions 56.00, 61.00, and 73.00 detected from 6.60 to $7.70 \mathrm{~min}$, (v) for butyl acetate, ions 57.00, 71.00, and 87.00 detected from 7.70 to $9.45 \mathrm{~min}$, (vi) for isoamyl acetate, ions 58.00, 70.00, and 88.00 detected from 9.45 to $13.10 \mathrm{~min}$, and (vii) for benzyl acetate, ions $63.00,107.00$, and 150.00 from 13.10 to $15.82 \mathrm{~min}$.

\section{Statistics}

Statistical analysis was performed with SigmaPlot v.14 using the two-tailed unpaired Student's $t$ test. 


\section{Supplementary information}

Supplementary information accompanies this paper at https://doi. org/10.1186/s13068-019-1563-z.

Additional file 1: Table S1. Summary of high cell density cultures of EcJW101, EcJW102, EcJW103, EcJW104, and EcJW105 with addition of glucose and various alcohols after $24 \mathrm{~h}$. The subscripts $\mathrm{i}$ and f are referred to the initial $(0 \mathrm{~h})$ and final $(24 \mathrm{~h})$ time of the culture, respectively. Table S2. Summary of titer, specific productivity, and yield of esters in high cell density cultures of EcJW101, EcJW102, EcJW103, EcJW104, and EcJW105 with addition of glucose and various alcohols after $24 \mathrm{~h}$. The acyl acetate and acyl lactate columns correspond to the acyl alcohols added. For example, with the exogenous addition of ethanol, the acyl acetate, and acyl lactate columns represent ethyl acetate, and ethyl lactate, respectively. Table S3. (A) Summary of high cell density cultures of EcDL002, EcJW201, and EcJW202 after $24 \mathrm{~h}$. (B) Summary of bioreactor batch fermentation of EcJW201 after $18 \mathrm{~h}$. The subscripts $\mathrm{i}$ and $\mathrm{f}$ are referred to the initial $(0 \mathrm{~h})$ and final ( $24 \mathrm{~h}$ ) time of the culture, respectively. Table S4. Summary of high cell density cultures of EcJW106-108 and EcJW203-208 with different concentrations of IPTG $(0.01,0.1$, or $1.0 \mathrm{mM})$ after $24 \mathrm{~h}$. The subscripts i and $\mathrm{f}$ are referred to the initial $(0 \mathrm{~h})$ and final $(24 \mathrm{~h})$ time of the culture, respectively. Table $\mathbf{S 5}$. Summary of titer, specific productivity, and yield of esters in high cell density cultures of EcJW106-108 and EcJW203-208 with different concentrations of IPTG $(0.01,0.1$, or $1.0 \mathrm{mM})$ after $24 \mathrm{~h}$. Table S6. Summary of high cell density cultures of EcJW209-212 with or without addition of ethanol (2 or $10 \mathrm{~g} / \mathrm{L}$ ) after $24 \mathrm{~h}$. (A) OD600, pH, glucose, lactate, and ethanol. (B) Titer, specific productivity, and yield of esters. The subscripts $i$ and fare referred to the initial $(0 \mathrm{~h})$ and final $(24 \mathrm{~h})$ time of the culture, respectively. Table S7. Summary of high cell density cultures of EcJW213-221 after $24 \mathrm{~h}$. (A) OD600, pH, glucose, lactate, and ethanol. (B) Titer, specific productivity, and yield of esters. The subscripts $\mathrm{i}$ and $\mathrm{f}$ are referred to the initial $(0 \mathrm{~h})$ and final $(24 \mathrm{~h})$ time of the culture, respectively. Table S8. Summary of high cell density cultures of EcJW109-117 after 24 h. (A) OD600, pH, glucose, lactate, and ethanol. (B) Titer, specific productivity, and yield of esters. The subscripts $i$ and $f$ are referred to the initial $(0 \mathrm{~h})$ and final $(24 \mathrm{~h})$ time of the culture, respectively.

Additional file 2: Figure S1. Expression of the recombinant enzymes in engineered E. coli strains. The positions corresponding to the overexpressed proteins are indicated by arrowheads. Lane $M$ represents protein ladder while lanes T, S, and I are referred to total, soluble, and insoluble proteins, respectively. (1) (3), Pyruvate-to-lactate ester module; (4) (5), Ethanol module; (6) (10), Isobutanol module. Protein sizes were predicted with their amino acids sequences. Figure S2. Effect of lactate esters on cell growth. (A) Specific growth rates of EcDL002 with or without addition of lactate esters. (B) logP values of characterized lactate esters. The values were obtained from http://www.thegoodscentscompany .com. (C-H) Growth curves of EcDL002 with or without addition of $(\mathbf{C})$ n-ethyl lactate (NEL), (D) n-propyl lactate (NPL), (E) n-butyl lactate (NBL), (F) $i$-butyl lactate (IBL), (G) $i$-amyl lactate (IAL), and (H) benzyl lactate (BZL). Figure S3. Design of (A) upstream module and (B) downstream module of the ethyl lactate pathway. The RBS Calculator v2.0 software was used to generate synthetic RBS sequences. For the upstream, four synthetic RBS sequences were generated with predicted translation initiation rates at 0.33 and 0.03 between the PAY 1 or PAY3 promoter and pdc start codon. For the downstream, six synthetic RBS sequences were generated with predicted translation initiation rates at 90,9000, and 90000 a.u. between the PT7 promoter and pct or VAAT start codon. Figure S4. (A) Correlation between ester production and the amount of added ethanol in high cell density cultures of EcJW209-212. (B) Correlation between ester production and the RBS strength for VAAT expression in high cell density culture of EcJW213-221.

\section{Abbreviations}

LdhA: lactate dehydrogenase; Pct: propionate CoA-transferase; AAT: alcohol acyltransferase; ATF1: alcohol acyltransferase from Saccharomyces cerevisiae; ATF2: alcohol acyltransferase from Saccharomyces cerevisiae; SAAT: alcohol acyltransferase from Fragaria ananassa; VAAT: alcohol acyltransferase from Fragaria vesca; AtfA: alcohol acyltransferase from Acinetobacter sp. ADP1; OD: optical density; DCW: dry cell weight; SDS-PAGE: sodium dodecyl sulfate-polyacrylamide gel electrophoresis; IPTG: isopropyl $\beta$-D-thiogalactopyranoside; MCS: multi-cloning site; RBS: ribosome binding site; au: arbitrary unit; HPLC: high-performance liquid chromatography; GC/MS: gas chromatography coupled with mass spectrometry; SIM: selected ion monitoring; DCM: dichloromethane; rpm: revolutions per minute; v/v: volume per volume; vvm: volume per volume per minute.

\section{Acknowledgements}

The authors would like to acknowledge the Center of Environmental Biotechnology at UTK for using the GC/MS instrument.

\section{Authors' contributions}

CTT conceived and supervised this study. JWL and CTT designed the experiments, analyzed the data, and drafted the manuscript. JWL performed the experiments. Both authors read and approved the final manuscript.

\section{Funding}

This research was financially supported in part by the NSF CAREER award (NSF\#1553250) and both the BioEnergy Science Center (BESC) and Center for Bioenergy Innovation (CBI), the U.S. Department of Energy (DOE) Bioenergy Research Centers funded by the Office of Biological and Environmental Research in the DOE Office of Science.

\section{Availability of supporting data}

Additional files 1 and 2 contain supporting data.

\section{Ethics approval and consent to participate}

Not applicable.

\section{Consent for publication}

All the authors consent for publication.

\section{Competing interests}

The authors declare that they have no competing interests.

\section{Author details}

${ }^{1}$ Bredesen Center for Interdisciplinary Research and Graduate Education, University of Tennessee, Knoxville, TN, USA. ${ }^{2}$ Center for Bioenergy Innovation, Oak Ridge National Laboratory, Oak Ridge, TN, USA. ${ }^{3}$ Department of Chemical and Biomolecular Engineering, University of Tennessee, 1512 Middle Dr., DO\#432, Knoxville, TN 37996, USA.

Received: 23 April 2019 Accepted: 7 September 2019

Published online: 20 September 2019

\section{References}

1. Kerton FM. Alternative solvents for green chemistry. Cambridge: RSC Pub; 2009.

2. Lancaster M. Green chemistry: an introductory text. Cambridge: Royal Society of Chemistry; 2002.

3. Aparicio S, Halajian S, Alcalde R, Garcia B, Leal JM. Liquid structure of ethyl lactate, pure and water mixed, as seen by dielectric spectroscopy, solvatochromic and thermophysical studies. Chem Phys Lett. 2008:454(1-3):49-55

4. Paul S, Pradhan K, Das AR. Ethyl lactate as a green solvent: a promising bio-compatible media for organic synthesis. Curr Green Chem. 2016;3(1):111-8.

5. Weissermel K, Arpe H-J. Industrial organic chemistry. 3rd ed. Weinheim: VCH Publishers Inc.; 1997.

6. Pereira CSM, Silva VMTM, Rodrigues AE. Ethyl lactate as a solvent: properties, applications and production processes - a review. Green Chem. 2011;13(10):2658-71.

7. Biddy MJ, Scarlata C, Kinchin C. Chemicals from biomass: a market assessment of bioproducts with near-term potential. Washington, D.C: National Renewable Energy Laboratory; 2016.

8. Gao C, Ma CQ, Xu P. Biotechnological routes based on lactic acid production from biomass. Biotechnol Adv. 2011:29(6):930-9. 
9. Hasegawa S, Azuma M, Takahashi K. Stabilization of enzyme activity during the esterification of lactic acid in hydrophobic ethers and ketones as reaction media that are miscible with lactic acid despite their high hydrophobicity. Enzyme Microb Technol. 2008;43(3):309-16.

10. Layton DS, Trinh CT. Engineering modular ester fermentative pathways in Escherichia coli. Metab Eng. 2014;26:77-88.

11. Rodriguez GM, Tashiro Y, Atsumi S. Expanding ester biosynthesis in Escherichia coli. Nat Chem Biol. 2014;10(4):259.

12. Tai YS, Xiong MY, Zhang KC. Engineered biosynthesis of medium-chain esters in Escherichia coli. Metab Eng. 2015;27:20-8.

13. Layton DS, Trinh CT. Expanding the modular ester fermentative pathways for combinatorial biosynthesis of esters from volatile organic acids. Biotechnol Bioeng. 2016;113(8):1764-76.

14. Wang J, Mahajani M, Jackson SL, Yang YP, Chen MY, Ferreira EM, Lin YH, Yan YJ. Engineering a bacterial platform for total biosynthesis of caffeic acid derived phenethyl esters and amides. Metab Eng. 2017;44:89-99.

15. Kruis AJ, Levisson M, Mars AE, van der Ploeg M, Daza FG, Ellena V, Kengen SWM, van der Oost J, Weusthuis RA. Ethyl acetate production by the elusive alcohol acetyltransferase from yeast. Metab Eng. 2017;41:92-101.

16. Layton DS, Trinh CT. Microbial synthesis of a branched-chain ester platform from organic waste carboxylates. Metab Eng Commun. 2016:3:245-51.

17. Guo DY, Pan H, Li X. Metabolic engineering of Escherichia coli for production of biodiesel from fatty alcohols and acetyl-CoA. Appl Microbiol Biot. 2015;99(18):7805-12.

18. Guo DY, Zhang LH, Kong SJ, Liu ZJ, Li X, Pan H. Metabolic engineering of Escherichia coli for production of 2-phenylethanol and 2-phenylethyl acetate from glucose. J Agric Food Chem. 2018;66(23):5886-91.

19. Liu ZJ, Zong Z, Chen ZJ, Xu QY, Shi Y, Li DS, Pan H, Guo DY. De novo biosynthesis of antimycobacterial agent geranylgeranyl acetate from glucose. Biochem Eng J. 2019;142:84-8.

20. Wu T, Li S, Zhang B, Bi C, Zhang X. Engineering Saccharomyces cerevisiae for the production of the valuable monoterpene ester geranyl acetate. Microb Cell Fact. 2018;17(1):85

21. Chacon MG, Marriott A, Kendrick EG, Styles MQ, Leak DJ. Esterification of geraniol as a strategy for increasing product titre and specificity in engineered Escherichia coli. Microb Cell Fact. 2019;18:105.

22. Guo DY, Kong SJ, Zhang LH, Pan H, Wang C, Liu ZJ. Biosynthesis of advanced biofuel farnesyl acetate using engineered Escherichia coli. Bioresour Technol. 2018;269:577-80.

23. Zhou S, Causey TB, Hasona A, Shanmugam KT, Ingram LO. Production of optically pure D-lactic acid in mineral salts medium by metabolically engineered Escherichia coli W3110. Appl Environ Microbiol. 2003;69(1):399-407.

24. Liu H, Kang J, Qi Q, Chen G. Production of lactate in Escherichia coli by redox regulation genetically and physiologically. Appl Biochem Biotechnol. 2011;164(2):162-9.

25. Chang DE, Jung HC, Rhee JS, Pan JG. Homofermentative production of D- or L-lactate in metabolically engineered Escherichia coli RR1. Appl Environ Microbiol. 1999;65(4):1384-9.

26. Niu DD, Tian KM, Prior BA, Wang M, Wang ZX, Lu FP, Singh S. Highly efficient L-lactate production using engineered Escherichia coli with dissimilar temperature optima for L-lactate formation and cell growth. Microb Cell Fact. 2014;13:78.

27. Chen XZ, Tian KM, Niu DD, Shen W, Algasan G, Singh S, Wang ZX. Efficient bioconversion of crude glycerol from biodiesel to optically pure D-lactate by metabolically engineered Escherichia coli. Green Chem. 2014;16(1):342-50.

28. Selmer T, Willanzheimer A, Hetzel M. Propionate CoA-transferase from Clostridium propionicum. Cloning of the gene and identification of glutamate 324 at the active site. Eur J Biochem 2002,269(1):372-80.

29. Taguchi S, Yamadaa M, Matsumoto K, Tajima K, Satoh Y, Munekata M, Ohno K, Kohda K, Shimamura T, Kambe H, et al. A microbial factory for lactate-based polyesters using a lactate-polymerizing enzyme. Proc Natl Acad Sci USA. 2008;105(45):17323-7.

30. Matsumoto K, Tobitani K, Aoki S, Song Y, Ooi T, Taguchi S. Improved production of poly(lactic acid)-like polyester based on metabolite analysis to address the rate-limiting step. AMB Express. 2014;4:83.

31. Ingram LO, Conway T, Clark DP, Sewell GW, Preston JF. Genetic-engineering of ethanol-production in Escherichia coli. Appl Environ Microb. 1987:53(10):2420-5.
32. Trinh CT, Unrean P Srienc F Minimal Escherichia coli cell for the most efficient production of ethanol from hexoses and pentoses. Appl Environ Microb. 2008;74(12):3634-43.

33. Choi YJ, Park JH, Kim TY, Lee SY. Metabolic engineering of Escherichia coli for the production of 1-propanol. Metab Eng. 2012;14(5):477-86.

34. Hanai T, Atsumi S, Liao JC. Engineered synthetic pathway for isopropanol production in Escherichia coli. Appl Environ Microb. 2007;73(24):7814-8.

35. Atsumi S, Cann AF, Connor MR, Shen CR, Smith KM, Brynildsen MP, Chou KJY, Hanai T, Liao JC. Metabolic engineering of Escherichia coli for 1-butanol production. Metab Eng. 2008;10(6):305-11.

36. Atsumi S, Wu TY, Eckl EM, Hawkins SD, Buelter T, Liao JC. Engineering the isobutanol biosynthetic pathway in Escherichia coli by comparison of three aldehyde reductase/alcohol dehydrogenase genes. Appl Microbiol Biotechnol. 2010;85(3):651-7

37. Tseng HC, Prather KLJ. Controlled biosynthesis of odd-chain fuels and chemicals via engineered modular metabolic pathways. Proc Natl Acad Sci USA. 2012;109(44):17925-30.

38. Connor MR, Liao JC. Engineering of an Escherichia coli strain for the production of 3-methyl-1-butanol. Appl Environ Microb. 2008;74(18):5769-75.

39. Pugh S, McKenna R, Halloum I, Nielsen DR. Engineering Escherichia coli for renewable benzyl alcohol production. Metab Eng Commun. 2015:2:39-45.

40. Sekar BS, Lukito BR, Li Z. Production of natural 2-phenylethanol from glucose or glycerol with coupled Escherichia coli strains expressing L-phenylalanine biosynthesis pathway and artificial biocascades. ACS Sustain Chem Eng. 2019;7(14):12231-9.

41. Kim TY, Lee SW, Oh MK. Biosynthesis of 2-phenylethanol from glucose with genetically engineered Kluyveromyces marxianus. Enzyme Microb Technol. 2014;61-62:44-7.

42. Stoveken T, Kalscheuer R, Malkus U, Reichelt R, Steinbuchel A. The wax ester synthase/acyl coenzyme A: diacylglycerol acyltransferase from Acinetobacter sp. strain ADP1: characterization of a novel type of acyltransferase. J Bacteriol. 2005;187(4):1369-76.

43. Wilbanks B, Trinh CT. Comprehensive characterization of toxicity of fermentative metabolites on microbial growth. Biotechnol Biofuels. 2017:10(1):262

44. Wu G, Yan Q, Jones JA, Tang YJJ, Fong SS, Koffas MAG. Metabolic burden: cornerstones in synthetic biology and metabolic engineering applications. Trends Biotechnol. 2016;34(8):652-64.

45. Schweiger G, Buckel W. On the dehydration of (R)-lactate in the fermentation of alanine to propionate by Clostridium propionicum. FEBS Lett. 1984;171(1):79-84.

46. Niu W, Guo J. Stereospecific microbial conversion of lactic acid into 1,2-propanediol. ACS Synth Biol. 2015;4(4):378-82.

47. Green MR, Sambrook J. Molecular cloning. A laboratory manual. New York: Cold Spring Harbor Laboratory Press; 2001.

48. Gibson D, Young L, Chuang R, Venter J, Hutchison C, Smith H. Enzymatic assembly of DNA molecules up to several hundred kilobases. Nat Methods. 2009:6:343-5.

49. Verstrepen KJ, Van Laere SDM, Vanderhaegen BMP, Derdelinckx G, Dufour JP, Pretorius IS, Winderickx J, Thevelein JM, Delvaux FR. Expression levels of the yeast alcohol acetyltransferase genes ATF1, Lg-ATF1, and ATF2 control the formation of a broad range of volatile esters. Appl Environ Microb. 2003;69(9):5228-37.

50. Aharoni A, Keizer LCP, Bouwmeester HJ, Sun Z, Alvarez-Huerta M, Verhoeven HA, Blaas J, van Houwelingen AMML, De Vos $\mathrm{RCH}$, van der Voet $\mathrm{H}$, et al. Identification of the SAAT gene involved in strawberry flavor biogenesis by use of DNA microarrays. Plant Cell. 2000;12(5):647-62.

51. Beekwilder J, Alvarez-Huerta M, Neef E, Verstappen FW, Bouwmeester HJ, Aharoni A. Functional characterization of enzymes forming volatile esters from strawberry and banana. Plant Physiol. 2004;135(4):1865-78.

52. Shi S, Valle-Rodriguez JO, Khoomrung S, Siewers V, Nielsen J. Functional expression and characterization of five wax ester synthases in Saccharomyces cerevisiae and their utility for biodiesel production. Biotechnol Biofuels. 2012;5:7.

53. Wu JJ, Du GC, Zhou JW, Chen J. Metabolic engineering of Escherichia coli for (2S)-pinocembrin production from glucose by a modular metabolic strategy. Metab Eng. 2013;16:48-55. 
54. Borujeni AE, Channarasappa AS, Salis HM. Translation rate is controlled by coupled trade-offs between site accessibility, selective RNA unfolding and sliding at upstream standby sites. Nucleic Acids Res. 2014;42(4):2646-59.

55. Salis HM, Mirsky EA, Voigt CA. Automated design of synthetic ribosome binding sites to control protein expression. Nat Biotechnol. 2009;27(10):946-U112.

56. Zhu Y, Eiteman MA, DeWitt K, Altman E. Homolactate fermentation by metabolically engineered Escherichia coli strains. Appl Environ Microbiol. 2007;73(2):456-64.

57. Trinh CT, Li J, Blanch HW, Clark DS. Redesigning Escherichia coli metabolism for anaerobic production of isobutanol. Appl Environ Microbiol. 2011;77(14):4894-904.
58. Wierzbicki M, Niraula N, Yarrabothula A, Layton DS, Trinh CT. Engineering an Escherichia coli platform to synthesize designer biodiesels. J Biotechnol. 2016:224:27-34.

\section{Publisher's Note}

Springer Nature remains neutral with regard to jurisdictional claims in published maps and institutional affiliations.
Ready to submit your research? Choose BMC and benefit from:

- fast, convenient online submission

- thorough peer review by experienced researchers in your field

- rapid publication on acceptance

- support for research data, including large and complex data types

- gold Open Access which fosters wider collaboration and increased citations

- maximum visibility for your research: over $100 \mathrm{M}$ website views per year

At BMC, research is always in progress.

Learn more biomedcentral.com/submissions 